\title{
Genomic features defining exonic variants that modulate splicing
}

\author{
Adam Woolfe ${ }^{1 *}$, James C Mullikin², Laura Elnitski ${ }^{*}$
}

\begin{abstract}
Background: Single point mutations at both synonymous and non-synonymous positions within exons can have severe effects on gene function through disruption of splicing. Predicting these mutations in silico purely from the genomic sequence is difficult due to an incomplete understanding of the multiple factors that may be responsible. In addition, little is known about which computational prediction approaches, such as those involving exonic splicing enhancers and exonic splicing silencers, are most informative.

Results: We assessed the features of single-nucleotide genomic variants verified to cause exon skipping and compared them to a large set of coding SNPs common in the human population, which are likely to have no effect on splicing. Our findings implicate a number of features important for their ability to discriminate spliceaffecting variants, including the naturally occurring density of exonic splicing enhancers and exonic splicing silencers of the exon and intronic environment, extensive changes in the number of predicted exonic splicing enhancers and exonic splicing silencers, proximity to the splice junctions and evolutionary constraint of the region surrounding the variant. By extending this approach to additional datasets, we also identified relevant features of variants that cause increased exon inclusion and ectopic splice site activation.

Conclusions: We identified a number of features that have statistically significant representation among exonic variants that modulate splicing. These analyses highlight putative mechanisms responsible for splicing outcome and emphasize the role of features important for exon definition. We developed a web-tool, Skippy, to score coding variants for these relevant splice-modulating features.
\end{abstract}

\section{Background}

The majority of genes in mammalian genomes are made up of multiple exons separated by much longer introns. To create a mature mRNA, exons must be identified accurately from within the transcript and then spliced together by removing the intervening introns. This process is carried out by a large complex of small nuclear RNAs and polypeptides known as the spliceosome. Disruption to the fidelity of splicing, particularly of exons that are constitutively spliced, can effectively inactivate a gene by creating unstable mRNAs and defective protein structure, or cause disease by disrupting the balance of expression of different splice isoforms [1]. The most important features for exon recognition are the splice junctions that define the boundaries of the exons, at which the spliceosome must assemble. Mutations at

\footnotetext{
*Correspondence: woolfea@mail.nih.gov; elnitski@mail.nih.gov

${ }^{1}$ Genomic Functional Analysis Section, National Human Genome Research Institute, National Institutes of Health, Rockville, Maryland 20892, USA
}

sites causing splicing abnormalities make up around $15 \%$ of all point mutations that result in human genetic disease [2]. However, this figure is likely to be a significant underestimate of the contribution of splicing in disease, as there is an increasing number of studies showing that mutations within both exons and introns, but outside of the canonical splice sites, can also disrupt splicing [3]. In particular, the ability of nonsense, missense and even synonymous (silent) mutations to cause exon skipping is often overlooked due to the strong association of exonic mutations solely with protein coding changes. Indeed, as the skipping of the exon can lead to the removal of an entire protein domain or degradation of the mRNA via nonsense-mediated decay, splice-affecting variants (including synonymous changes) are much more deleterious than most missense mutations that substitute a single amino acid. Similarly, exonic variants can also result in deleterious effects by activating a de novo (that is, not pre-existing) ectopic 
splice site, which is then used in preference to the natural splice site, shortening the exon. A well-known example of this is a synonymous mutation in exon 11 of the human $L M N A$ gene that creates a 5' ectopic splice site. This shortens the protein sequence through frameshift, and causes the rare premature aging disorder Hutchinson-Gilford progeria [4].

The mechanism by which these internal exonic mutations exert their effect is still not fully understood, but they are most commonly associated with changes in regulatory elements within the exon that are important for exon definition. The spliceosome must distinguish genuine splice sites from a collection of sequences in the intron that resemble them but are never used (known as pseudo splice-sites). Therefore, correct exon recognition requires additional auxiliary signals present both within the exon and in the introns, as canonical splice sites are not sufficient to define the proper splice sites. These regulatory sequences, important in both constitutive and alternative splicing, can be broadly defined by their intergenic location and their effects on splicing. Those located within the exon and promoting exon inclusion are referred to as exonic splicing enhancers (ESEs) and those inhibiting exon inclusion are referred to as exonic splicing silencers (ESSs). Similarly those located in the intron are referred to as intronic splicing enhancers and intronic splicing silencers, although these are more commonly associated with specifying alternative splicing [5] or splicing of non-canonical introns [6].

Although identification and characterization of the complement of proteins that bind specific exonic enhancer and silencer elements is far from complete, most enhancer sequences within exons have been found to bind members of the serine/arginine-rich (SR) protein family, while many silencing elements are bound by members of the heterogeneous ribonuclearprotein (hnRNP) family [7]. ESE-bound SR proteins promote exon definition by directly recruiting and stabilizing the splicing machinery through protein-protein interactions [8] and/or antagonizing the function of nearby silencer elements [9]. Silencers are not as well characterized as enhancers, but ESS-bound hnRNPs are thought to mediate silencing through direct antagonism of the splicing machinery or by direct competition for overlapping enhancer-binding sites. The intrinsic strength by which the splice sites are recognized by the spliceosome as well as the antagonistic dynamics of proteins binding ESEs and ESSs control much of exon recognition and alternative splicing. It is therefore not surprising that exonic splicing regulatory sequences (ESRs) are now increasingly recognized as a major target and a common mechanism for disease-causing mutations leading to exon skipping in functionally diverse genes. Examples of disease mutations reported to destroy ESE motifs and cause exon skipping include those in the $B R C A 1$ [10], SMN1/2 [11], PDHA1 [12] and GH [13] genes.

Given the critical role of these sequences in exon splicing, significant research efforts are focused on identifying the complement of ESE and ESS binding sites involved in constitutive splicing. The assortment of enhancer and silencer sequences recognized by known splicing factors is considerable [3]. This suggests that ESRs may represent numerous functionally distinct classes, or may be recognized in a degenerate fashion. This 'fuzzy' definition of ESRs has meant that their precise characterization has proved challenging. A large group of existing ESE/ESS datasets has been identified either experimentally $[14,15]$ or through the use of computational approaches followed by some form of experimental verification of a subset of predictions [16-19] (for an overview see Table 1 and [20]). The motifs defined in each dataset are commonly represented as hexamers or octomers, or encoded as position weight matrices analogous to transcription factor binding sites. Motifs predicted by these approaches are partially overlapping, but also yield certain proportions that are unique or even contradictory. Recent studies have also suggested that both global and local RNA secondary structure may also play a role in the recognition and activity of splicing regulatory motifs in certain cases $[21,22]$. Despite our access to these varied splicing regulatory datasets, the question of whether they are effective in detecting the appropriate splicing regulatory changes associated with splice modulating variants has yet to be systematically assessed.

The development of high throughput sequencing technologies provides an unprecedented opportunity to identify disease alleles associated with both common and rare disorders. In the likelihood that exonic spliceaffecting mutations are a commonly overlooked phenomena in disease and transcript variation, it is important to identify the genomic features most relevant in characterizing novel splice-affecting genome variants (SAVs). We performed a comparative analysis using sets of experimentally verified SAVs against SNPs common in the human population, the majority of which are likely to be splicing-neutral. Comparative analysis of SNP datasets is a powerful approach to highlight characteristics that define disruptive sequence variants. A similar approach has been employed previously to predict SNPs affecting transcriptional cis-regulation [23] as well as to measure selective pressure on genomic elements such as conserved non-coding sequences [24] and splicing enhancers [25]. Here, we focused our main analyses on the most prevalent and least characterized SAVs, those that cause exon skipping, using a battery of bioinformatics approaches as well as a systematic comparison of all currently available ESE/ESS datasets, to identify 
Table 1 Exonic splicing regulatory elements datasets used in this study

\begin{tabular}{|c|c|c|c|}
\hline $\begin{array}{l}\text { ESR } \\
\text { dataset }\end{array}$ & Format & Method & Reference \\
\hline ESEFinder & 4 ESE PWMs & $\begin{array}{l}\text { Set of four experimentally derived ESE binding site matrices for four SR proteins (SF2, SC35, SRp40, } \\
\text { SRp55) identified by an in vitro SELEX approach with specific SR protein complementation }\end{array}$ & [14] \\
\hline $\begin{array}{l}\text { Fas- (hex3) } \\
\text { ESS }\end{array}$ & 176 ESS hexamers & $\begin{array}{l}\text { Set of experimentally derived ESSs identified in vivo through cloning of random decamers into } \\
\text { fluorescence activated minigene reporter by selecting those sequences that cause exon skipping. } \\
\text { Unique candidates were clustered and represented by non-degenerate hexamers }\end{array}$ & [15] \\
\hline $\begin{array}{l}\text { RESCUE- } \\
\text { ESE }\end{array}$ & 238 ESE hexamers & $\begin{array}{l}\text { Set of putative ESEs derived from overrepresented hexamer motifs in exons versus introns and exons } \\
\text { with weak splice sites versus exons with strong splice sites }\end{array}$ & [17] \\
\hline PESX & $\begin{array}{l}2,096 \text { ESE/974 ESS } \\
\text { octomers }\end{array}$ & $\begin{array}{l}\text { Set of putative ESES (PESE) and ESSS (PESS) overrepresented and underrepresented in internal non- } \\
\text { coding exons versus unspliced pseudoexons and } 5^{\prime} \text { UTRs of intronless genes }\end{array}$ & [18] \\
\hline $\mathrm{NI}-\mathrm{ESR}$ & $\begin{array}{l}979 \text { ESE/496 ESS } \\
\text { hexamers }\end{array}$ & $\begin{array}{l}\text { Uses the neighborhood inference (NI) algorithm to identify new candidate ESEs and ESSs using a set } \\
\text { of previously identified ESES/ESSs. The NI algorithm searches the sequence neighborhood of a } \\
\text { particular hexamer and scores it by whether the surrounding sequences contain mostly known ESES, } \\
\text { ESSs or neither. Predicted candidates were verified by cross-validation and a subset was } \\
\text { experimentally validated }\end{array}$ & [19] \\
\hline Ast-ESR & 285 hexamers & $\begin{array}{l}\text { Motifs based on computational analysis of overrepresented and conserved dicodons in orthologous } \\
\text { human-mouse exons. Putative ESRs are not labeled as ESEs or ESSs as a number were found to act as } \\
\text { both enhancers and silencers in minigene assays depending on sequence context. }\end{array}$ & [16] \\
\hline $\begin{array}{l}\text { Composite- } \\
\text { ESR }\end{array}$ & $\begin{array}{l}400 \text { ESE/217 ESS } \\
\text { hexamers }\end{array}$ & Combined set of ESE/ESS based on RESCUE-ESE, PESE, PESS and Fas-ESS datasets & [60] \\
\hline
\end{tabular}

PWM, position weight matrix.

the features of these SAVs and their exonic/intronic environment that are most likely to be predictive for exon skipping events. Extending this analysis, we also identified relevant features associated with SAVs causing increased exon inclusion and ectopic splice site creation. Combined, these features are useful to predict the probability of novel splice-modulatory events and are made available through a web server.

\section{Results}

To identify features associated with exon skipping SAVs, we collated a set of experimentally verified variants in the human genome that independently cause exon skipping from extensive literature searches and the Alternative Splicing Mutation Database [26]. We excluded all variants from this list that may affect splicing through other well-defined mechanisms, such as nonsense-mediated exon skipping or disruption of canonical splice sites (see Materials and methods). A total of 87 variants were identified (currently the largest dataset of its kind), and their genomic positions mapped back onto the human genome (hg18). As the majority of analyses in this paper involve exon-skipping SAVs, we refer to these variants simply as 'SAVs', unless otherwise indicated. This set is made up of 32 synonymous and 55 missense SAVs distributed across 43 genes and 47 individual exons (Additional file 1). Of these, $87 \%$ (41) were constitutively spliced and $13 \%$ (6) were alternatively spliced cassette exons.

In addition to known SAVs, a set of spicing-neutral variants (that is, that have little or no effect on exon splicing) served as a standard for comparison. Although no large-scale set of experimentally verified splice-neutral variants has been published, through literature searches we identified a set of 80 variants that were tested in mini-gene splicing assays and were found to have no effect on splicing (Additional file 2). Unfortunately, around half of these derive from artificial mutagenesis studies, and may therefore include certain artificial biases. As an alternative, since exonskipping events are likely to be largely deleterious, we exploited the principle that SAVs will be largely absent from polymorphisms common in the human population. Phase II HapMap SNPs represent both a high quality and extensive genome-wide set of human polymorphisms, as they have been genotyped for 270 individuals in four populations [27]. From this set of 3.1 million SNPs, we took all SNPs that fell in internal (that is, spliced) coding exons that were polymorphic in at least one individual and filtered them in the same way as SAVs (see Materials and methods). In addition, we only retained SNPs where we could determine the ancestral and derived allele with high confidence by utilizing orthologous positions in the chimp and macaque genomes. This approach allowed us to make an assumption of the allele directionality, which was important for detecting loss or gain of splice regulatory elements. The resulting dataset contained 15,547 SNPs with roughly equal numbers of synonymous and missense alleles (7,922 and 7,625, respectively). These SNPs fell within 13,163 individual exons from 7,038 genes. For ease of reference, we refer to this set of HapMap SNPs as 'hSNPs'.

Using these sets of variants, we carried out comparative analyses to identify the features that discriminate 
SAVs from hSNPs, which can be described at the sequence level (such as changes in the underlying splicing regulatory sequences and physical location within exons), or at the exon level (to predispose an exon to exon-skipping events) to enable a predictive framework for uncharacterized variants.

\section{Variant-based features}

\section{Changes in exonic splicing regulatory sequences}

Our systematic assessment of all seven currently available ESR datasets examined their ability to identify splice-regulatory elements in the verified SAV sequences. This approach assessed the types of motifaltering changes associated with SAVs and provided benchmarking of the seven ESR collections (Table 1) to determine which most strongly differentiated real spliceaffecting variants from common polymorphisms. Of these seven sets, two contain ESEs (ESEFinder and RESCUE-ESE), one represents ESSs (Fas-ESS) and the remaining four sets contain both ESEs and ESSs (PESX, NI-ESR, Ast-ESR and Composite-ESR). For both SAV and hSNP sequences we measured three possible types of changes in the ancestral versus derived allele (or wild type versus disease allele) as a result of the variant: ESR loss, ESR gain and ESR alteration (see Materials and methods).

We first examined whether the proportion of SAVs with a particular type of ESR change was significantly different from that of hSNPs. Our comparative analyses identified two significant changes associated with variants that cause exon skipping: the gain of sequences defined as ESSs and the loss of sequences defined as ESEs (Figure 1; Additional file 3). Of these, we found that ESS gains had stronger discriminatory power than ESE losses. All the ESS datasets identified a significantly greater proportion of SAVs causing gains of ESSs. In contrast, results for ESE losses were split. NI-ESE, RESCUE-ESE and Comp-ESE showed a moderate but

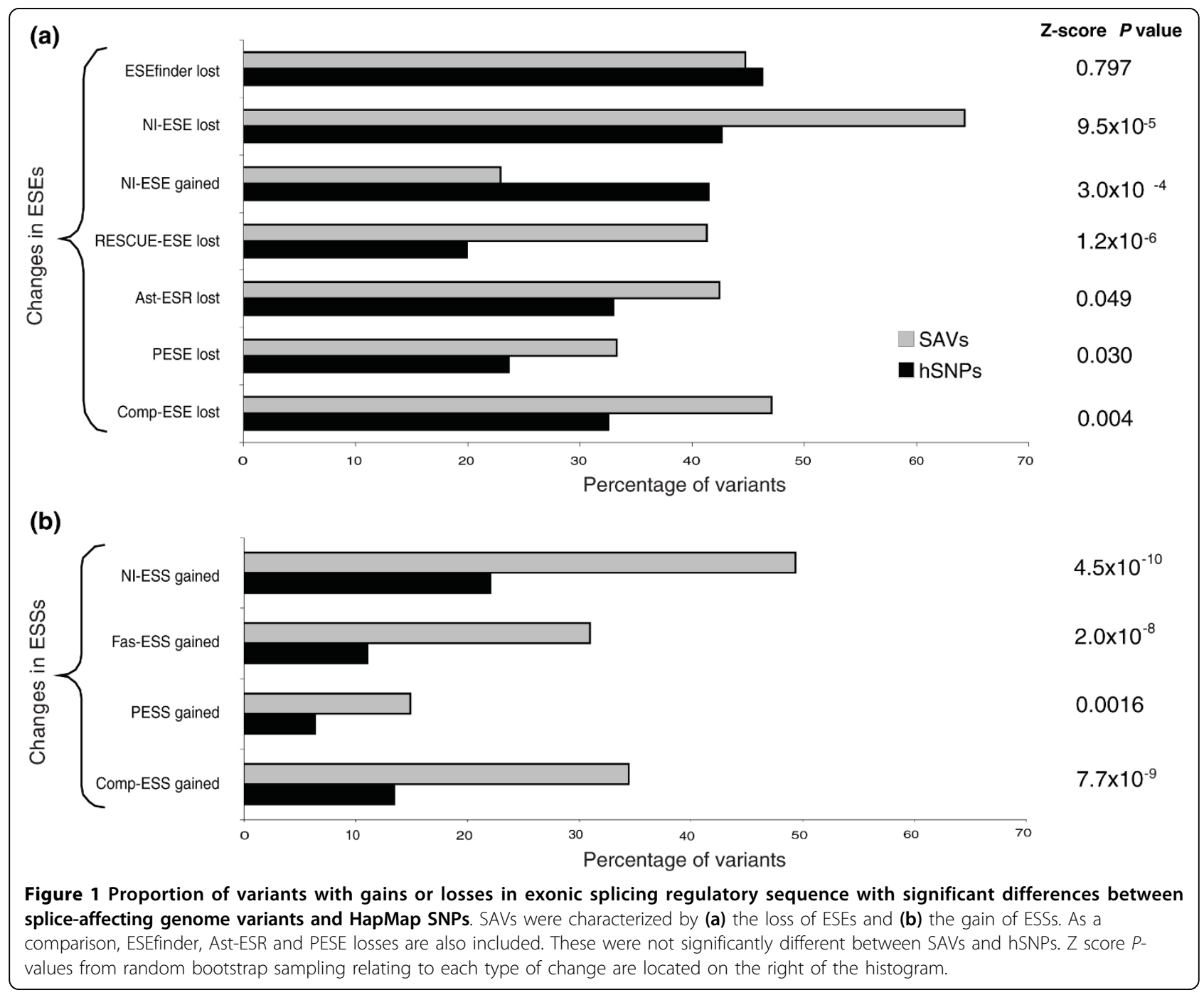


significantly greater proportion of ESE losses in SAVs than hSNPs. Losses of ESEfinder motifs were roughly equal between SAVs and hSNPs, both as a group of motifs and individually (Figure 1; Additional file 3). Nevertheless, we hypothesized that because the threshold set for each ESEFinder binding site is somewhat arbitrary, single base changes that cause a binding site to be 'lost' may not be functionally equivalent and that changes in certain positions may be less tolerated than others. We found one position in each binding matrix that occurred at significantly higher numbers in SAVs compared to hSNPs (by $\chi^{2}$ test, $P<0.05$; Additional file 4). Hence, there may be different functional constraints acting along the binding sites that are not properly captured by the default scoring thresholds and the position weight matrix scores as currently employed. Ast-ESRs, while not explicitly defined as ESEs or ESSs, showed no significant difference between variant groups for losses, alterations or gains [16]. Consistent with the direction of the previous ESR changes, SAVs were also significantly diminished for gains of ESEs using the NI-ESE dataset (Figure 1; Additional file 3).

\section{The extent of ESR changes further differentiates SAVs from hSNPS}

We investigated whether SAVs are further distinguished by the cumulative extent of the ESE losses and ESS gains. Many of the sets of putative ESRs are represented as hexamers (for example, RESCUE-ESE, NI-ESRs, PESXs, and so on), either because this is often the size of a single protein-binding site (for example, the GAAGAA ESE [28]), or because they are a reduced representation of larger binding sites. Because point variants may modulate several overlapping binding sites simultaneously, those affecting larger numbers of predicted sites are more likely to have significant impact, for which we assessed predictive power. The results showed that in all ESR sets except ESEfinder, numbers of ESS gains and ESE losses were much greater in SAVs than hSNPs (Additional file 3). We saw the greatest separation from hSNPs using NI-ESSs gains (98 gains in SAVs versus a mean of 32 in hSNPs, Z-score $P=1.92 \times 10^{-17}$ ) and NI-ESEs losses (138 losses in SAVs versus a mean of 69 in hSNPs, Z-score $P=2.68 \times 10^{-10}$ ), although RESCUE-ESE, Fas-ESS and Composite-ESR also give good, strongly statistically significant separations, despite the much smaller size of these datasets compared to NIESRs (Table 1).

For NI-ESR, losses or gains of two or more motifs were prevalent, with the divergence between SAVs and hSNPs becoming larger as the total number of occurrences increased (Figure 2a, b). When the extent of ESS gains and ESE losses were combined as a total number of changes, $46 \%$ of SAVs had four or more such changes compared to only $9 \%$ for hSNPs (Figure 2c). Furthermore, we compared the set of 80 experimentally verified splice-neutral variants against the hSNP dataset and found that no category of ESR change was significantly different (Additional file 3). This supports our assumption that hSNPs act as an appropriate proxy for splice-neutral variants and confirms that significant ESR differences are detectable between spliceaffecting and splicing-neutral datasets.

Finally, using a recently established computational method [22], we investigated whether taking local RNA secondary structure into consideration improved the ability to distinguish functionally relevant ESR changes in SAVs from those in hSNPs. We found little evidence that local RNA secondary structure, as implemented by this method, improved our ability to differentiate these two datasets further (see Additional file 5 for methods and results).

\section{Splice-altering sequence changes are under negative selection in common SNPs}

In the previous comparative analyses, we assumed that the differential signal in ESR changes between SAVs and hSNPs was a composite consequence of both functional ESR changes in SAVs and selective pressure to avoid those changes in common hSNPs [25]. To test this assumption, we investigated whether the proportion of each type of ESR change in SAVs and hSNPs, using the NI-ESR dataset, would differ when compared to an 'expected' neutral distribution created through permutation (see Materials and methods). This permuted distribution represents what we would expect if variants occurred randomly under no selective pressure for splicing. We found that while hSNPs followed the expected distribution closely for many of the changes, SAVs had almost two-fold higher proportions of ESS gains and ESE losses (Figure 3), confirming that these types of changes were a non-random, characteristic property of SAVs. Moreover, the highly significant difference in ESS gains between SAVs and hSNPs can be further explained by a significant reduction for this type of change in hSNPs compared to the expected distribution (5.6\% of changes in hSNPs versus $8.3 \%$ under neutrality, $\chi^{2}$ test $P=1.7 \times$ $10^{-8}$ ), suggesting negative selection against the gain of silencers in common variants. We also identified a fivefold increase in the proportion of variants that cause direct changes from an ESE to an ESS in SAVs compared to both the expected and hSNP distributions $(4.1 \%$ of changes in SAVs versus $0.8 \%$ under neutrality/hSNPs, $\chi^{2}$ test $P=3.8 \times 10^{-12}$; Figure 3), indicating that this type of change represents a strong indicator of splice-affecting changes.

\section{Significant ESR changes in variants that increase exon inclusion}

We carried out the same comparative analysis against hSNPs using a smaller set of 20 exonic variants that have been experimentally verified to cause increased 

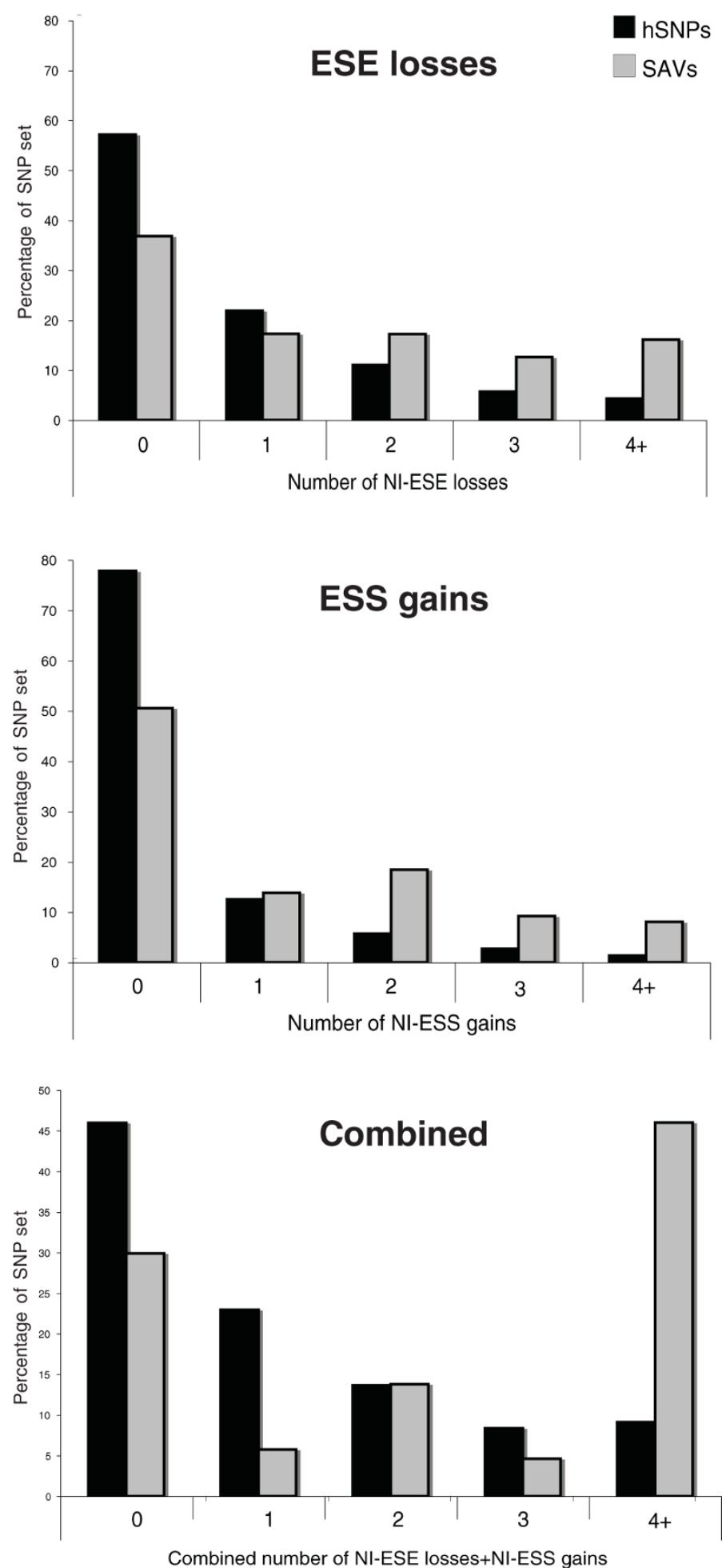

Figure 2 Splice-affecting genome variants are characterized by losses of large numbers of NI-ESEs and the gain of large numbers of NI-ESSs, often in combination. For both ESE losses and ESS gains, the proportion of SAVs with changes of two or more were significantly greater compared to hSNPs. Combinations of ESE losses and ESS gains, as opposed to each occurring independently, are highly enriched in SAVs compared to hSNPs (bottom graph). 


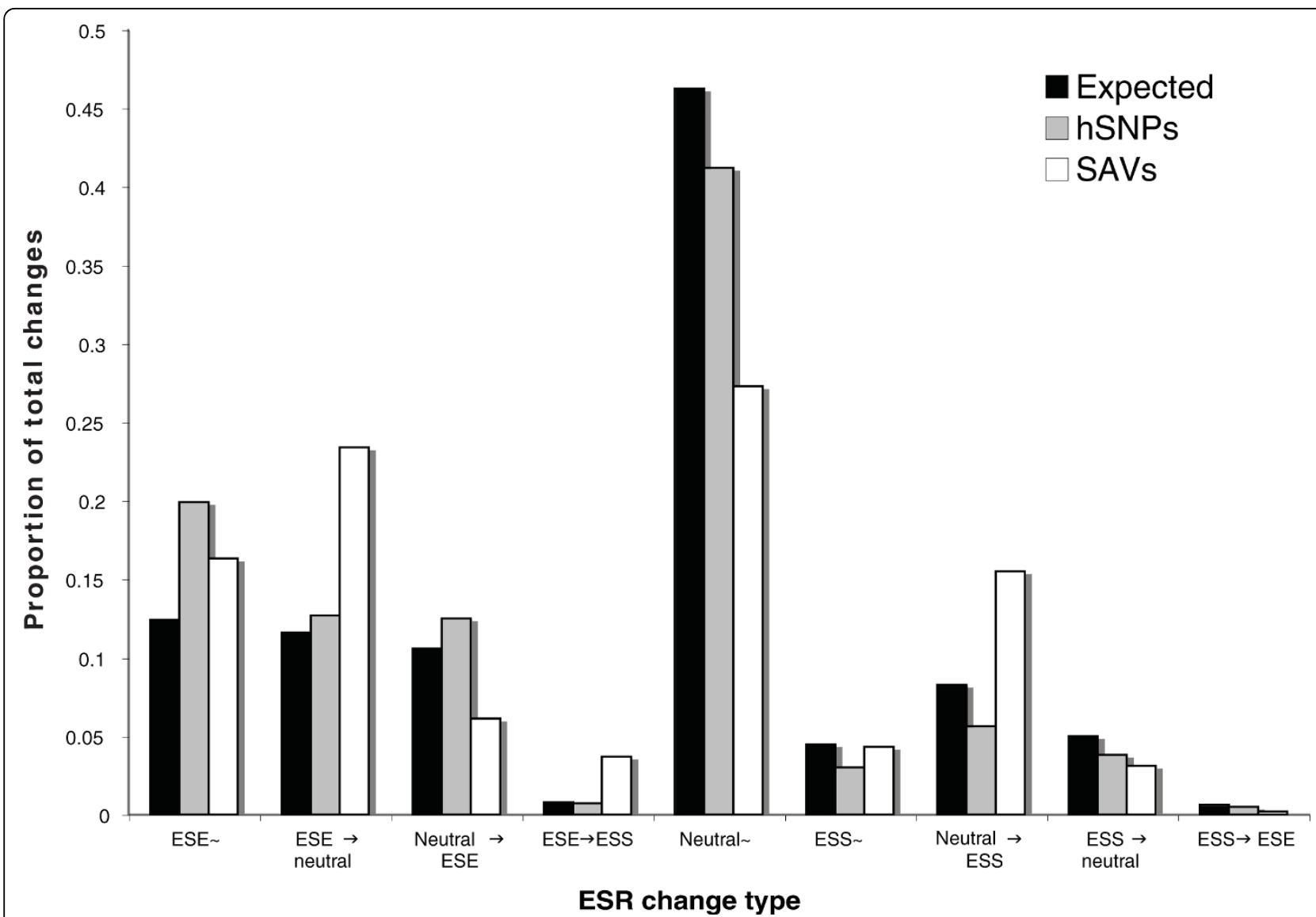

Figure 3 Distribution of specific types of NI-ESR changes for SAVs and hSNPs compared to neutral expectation. The tilde symbol $(\sim)$ signifies an alteration where the hexamer is designated an ESE, neutral or ESS in both the wild-type and variant sequences. The arrow represents the direction of the change as a consequence of the change between wild type and variant hexamer. The neutral expected distribution reflects the underlying probability of each type of change given the ESE/ESS distribution among NI hexamers and the genome-wide nucleotide substitution bias in coding regions.

exon inclusion (Additional file 6). Although lacking some of the statistical power of the larger exon skipping SAV set, we found that these variants were significantly enriched for ESSs losses (21 losses versus a mean of 5 in hSNPs, empirical $P=1 \times 10^{-4}$; Additional file 3). They also exhibited greater numbers of ESE gains (25 gains versus a mean of 15 in hSNPs, empirical $P=0.034)$ and lower numbers of ESE losses (5 losses versus a mean of 16 in hSNPs, empirical $P=$ 0.0097). These changes were the opposite of the changes caused by skipping SAVs and consistent with regulatory changes expected to increase exon definition. These results highlighted the antagonistic interplay between ESEs and ESSs in stabilizing or destabilizing exonic splicing.

\section{Proximity to exon boundaries}

Previous studies have shown that a number of exonic characteristics are affected by proximity to the exon junction, including ESE density [25], evolutionary constraint [16,29] and codon bias [30]. Although circumstantial, this evidence supports the view that the boundaries of exons contain regulatory 'hotspots' that may be more critical to splicing than centralized regions. To investigate whether SAVs are more likely to be disruptive if located preferentially in these hotspot regions, we divided all SAV exons and HapMap exons into six equal parts and binned the SAV or hSNP variants according to their locations. Figure 4 shows that hSNPs were distributed roughly equally across the exons, with a small depletion at exon boundaries, whereas SAVs were enriched close to the exon boundaries and depleted towards the center $(46 \%$ of SAVs located at the peripheral sections of exons versus $28.5 \%$ of hSNPs, $P=0.005$ ). Nevertheless, over a quarter of the SAVs are located within the central sections of the exon, suggesting that while variants located at the peripheries of the exon are likely to have the greatest effect on splicing, other elements important for splicing may be found at positions across the exon, but not with discriminatory power for this analysis. 


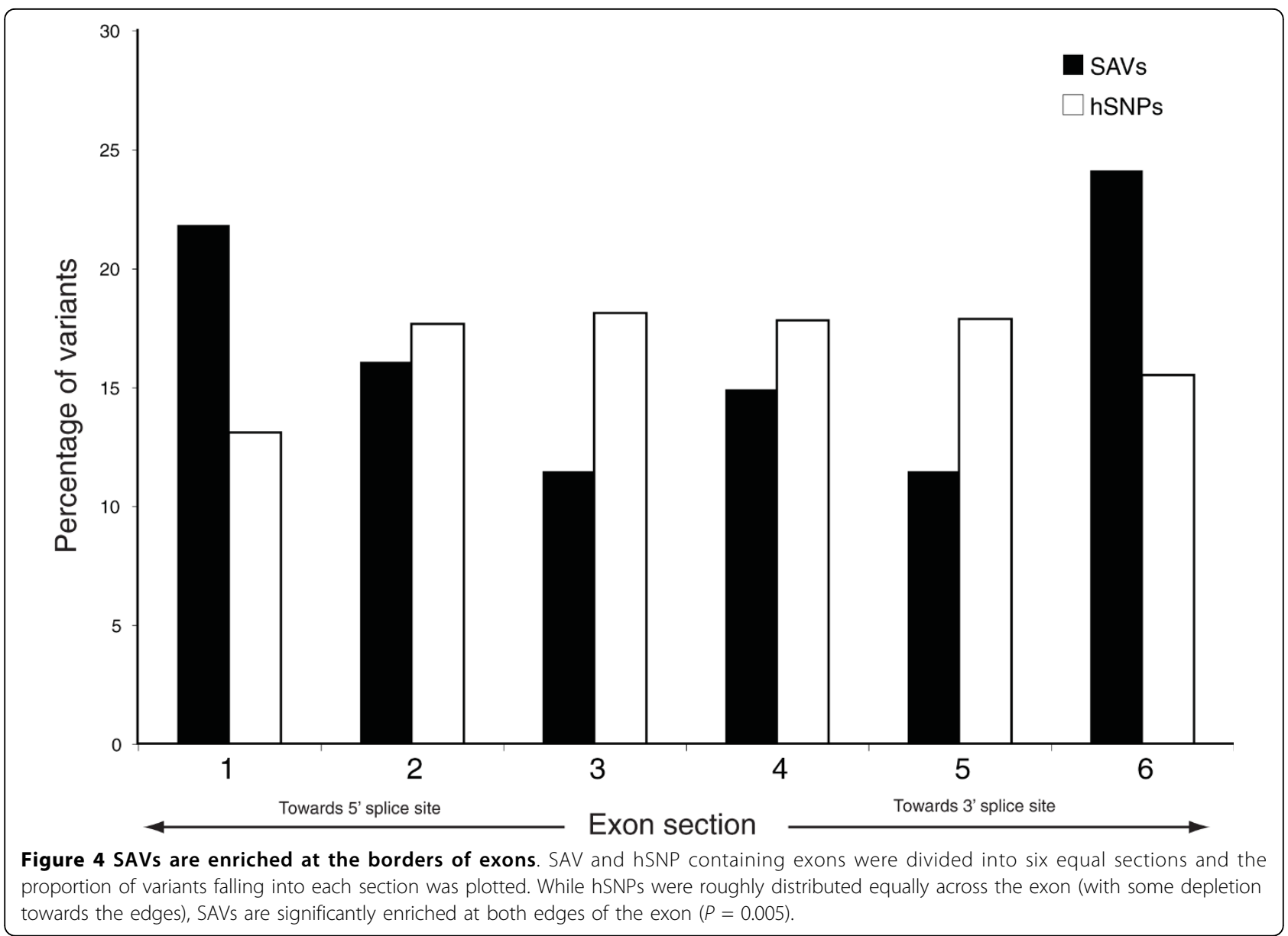

\section{Regulatory evolutionary constraint of SAV regions}

The availability of multiple sequenced mammalian genomes provides the opportunity for evolutionary comparisons of functional constraint across related species. Splicing patterns and exonic splicing regulatory elements are generally conserved across mammals [31]. Therefore, sequences important for splicing should be detectable by greater evolutionary sequence conservation; a case that is proven for intronic factors [32]. We hypothesized that the regions surrounding SAVs should be under greater evolutionary constraint than regions surrounding neutral variants. However, within coding exons, the constraint on the sequence due to splicing has to be decoupled from pre-existing protein-coding constraint. One solution is to measure conservation at synonymous codon positions, which are normally considered to be neutrally evolving. Several studies have demonstrated that ESRs increase selective constraint on synonymous positions $[16,33]$. An extreme example is the ultra-conservation of coding sequences that are associated with auto-regulatory alternative splicing of 'poison exons' in SR proteins [34].
To score regulatory constraint in coding regions, we created an expectation-based scoring matrix for each of the 192 positions of the genetic code. The scores were inversely proportional to conservation levels in genomewide human/mouse/rat/dog DNA multiple alignments (see Materials and methods). By using a scoring scheme based on real evolutionary data, the scoring matrix not only preferentially scores synonymous over non-synonymous positions, but also incorporates other influences, such as codon bias and hypermutability. For example, the highest scores in the matrix are at synonymous positions in hypermutable CpGs (that is, TCG, ACG, CCG and $\mathrm{GCG}$ ) as these are the least conserved coding positions genome-wide (Figure 5a). Using this scoring matrix, we calculated regulatory constraint (RC) scores in localized coding regions, representing all possible hexamer positions surrounding a variant, for all SAVs and hSNPs (Figure 5b) and compared the mean RC scores of all non-overlapping regions for each set. Results showed that sequences containing SAVs had significantly higher mean conservation scores than a random sampled distribution of hSNPs (1.583 versus a 


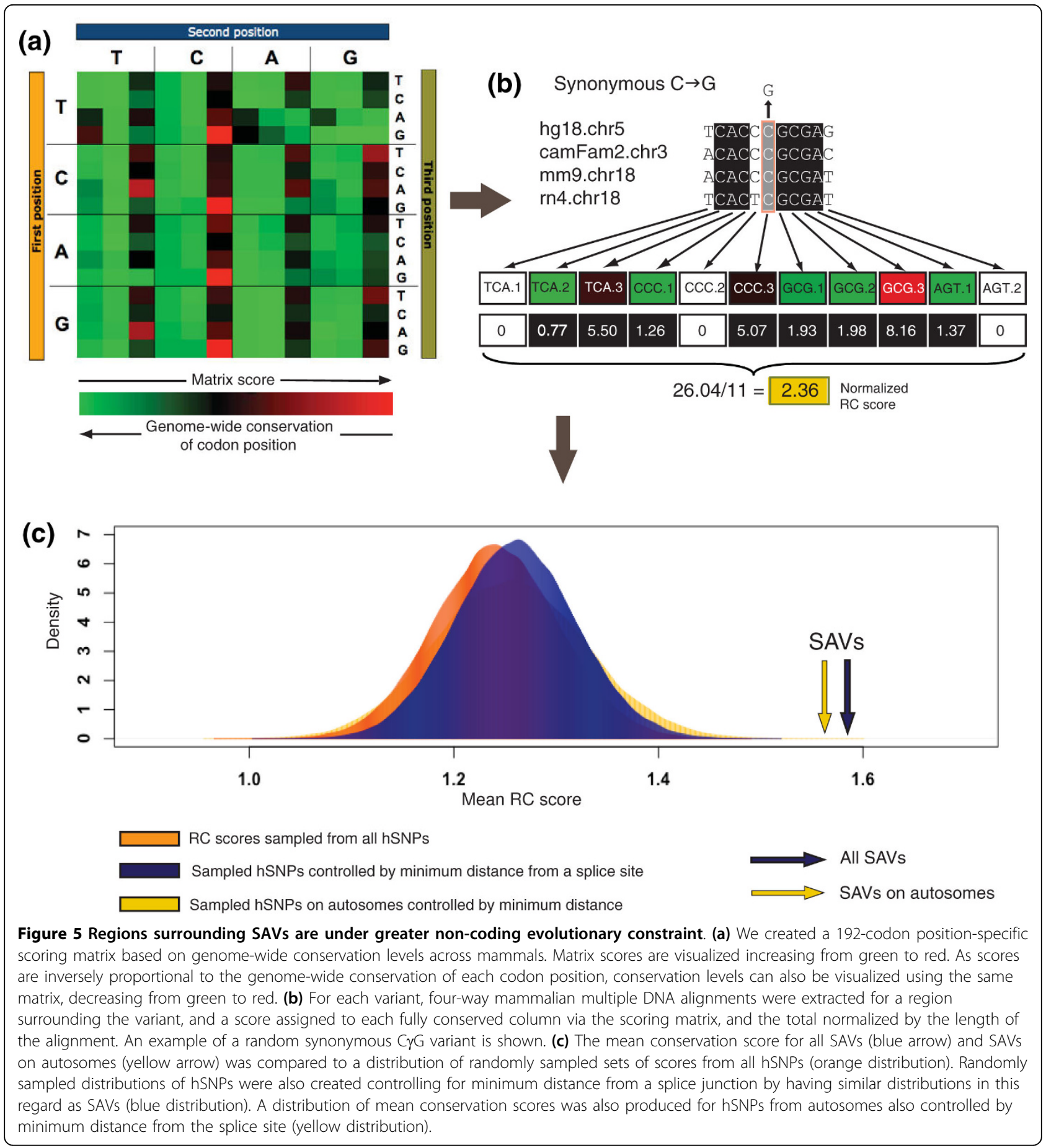

mean of 1.233 in hSNPs, $\mathrm{Z}$ score $P=5.71 \times 10^{-9}$; Figure $5 c$, orange distribution).

We addressed a variety of sources of bias that could confound the outcome of the conservation analysis. For example, rates of synonymous and non-synonymous substitutions decrease close to splice junctions $[29,30]$. Data from hSNPs confirmed this result by showing that the $\mathrm{RC}$ scores were negatively correlated with distance from the splice junction (Additional file 7). However, since SAVs are enriched close to splice junctions, we repeated the analysis choosing hSNPs with similar distances from the splice junction as those in the SAV set. This shifted the hSNP distribution to greater mean RC scores (Figure 5c, blue distribution), but the difference with SAVs remained highly significant $(1.583$ versus a mean of 1.266 in hSNPs, $Z$ score $P=1.92 \times 10^{-8}$ ). 
A second potentially significant source of bias was due to SAVs on the X chromosome contributing $35 \%$ of the variant set, compared to just $1.38 \%$ of the hSNP set. Prior SNP analyses identified the $\mathrm{X}$ chromosome as having lower rates of heterozygosity than autosomes [27], and human-mouse comparisons showed that genes on this chromosome were under greater evolutionary selection [35]. It was possible, therefore, that the prevalence of SAVs from the $\mathrm{X}$ chromosome contributed to the significantly higher conservation scores. We found that mean RC scores for hSNPs on the X chromosome were significantly higher than for other chromosomes (1.34 versus 1.24 , Kolmogorov-Smirnov (K-S) test $P=0.008$ ). Similarly, SAVs on the X chromosome had a higher mean RC score than SAVs on other chromosomes but the difference was not statistically significant (1.57 versus $1.67, \mathrm{~K}-\mathrm{S}$ test $P=0.33$ ) due to small sample sizes. We therefore repeated the analysis using only SAVs and $\mathrm{hSNPs}$ on autosomes (also controlling for distance from the splice junction; Figure 5c, yellow distribution). The difference in mean RC scores was further decreased but nevertheless remained highly significant ( 1.55 versus 1.25 , $\mathrm{Z}$ score $P=1.28 \times 10^{-5}$ ). Therefore, the predominance of SAVs from the $X$ chromosome was not sufficient to explain the greater regulatory constraint surrounding SAVs.

We also examined whether SAV exons were more highly conserved than HapMap exons. We compared percent-identity from four-way multiple alignments, across entire exons or within non-synonymous positions of exons, excluding the $\mathrm{X}$ chromosome. No significant differences were found in mean percent-identities in non-synonymous positions (89\% in SAV exons versus $88.7 \%$ in hSNP exons, $\mathrm{Z}$ score $P=0.122$ ) or overall (77\% in SAV exons versus $75 \%$ in hSNP exons, $Z$ score $P=0.063)$. Furthermore, similar results were obtained using HapMap exons of all sizes, or those that closely resembled the size distribution of SAV exons. By controlling for alternative sources of constraint we concluded SAVs occur in regions of exons that are under greater non-coding constraint, indicative of negative selection for important function.

\section{Exonic environment}

We addressed features associated with exon definition to test whether exons containing SAVs (which we will term 'SAV exons') are significantly different in these aspects from exons containing hSNPs (termed 'HapMap exons') or from exons in general, indicative of a pre-existing weakness or predisposition to the effects of SAVs.

\section{Exon size}

A comparison of exon lengths between SAV and HapMap exons showed that SAV exons were significantly smaller $($ mean $=125.1 \mathrm{bp}$ versus $197.8 \mathrm{bp}, \mathrm{K}-\mathrm{S}$ test $P=$ $\left.1.269 \times 10^{-7}\right)$. However, further comparison of the SAV exons to internal exons from the Hollywood exon annotation database [36] showed that both the mean (125 bp versus $136 \mathrm{bp}, P=0.39$ ) and median (112 bp versus 120 bp, $P=0.051$ ) values of the SAV exons, although lower, were not statistically different in a randomized bootstrap analysis (see Materials and methods). When compared directly to constitutive Hollywood exons, HapMap exons were significantly larger (K-S test $P<2.2 \times 10^{-16}$ ). We examined the potentially confounding problem of larger HapMap exons through simulation analyses and showed that the probability of an exon containing a SNP increased as exon length increased (see Materials and methods). The simulated exons with SNPs had the same length distribution as HapMap exons (Additional file 8). We therefore controlled for equivalent exon size in all subsequent analyses.

\section{Splice site strengths}

Signals critical for exon definition are the 5' and 3' splice sites and branch point. The strength of these signals may influence whether an exon is constitutively or alternatively spliced, creating conditional dependency on ESEs and vulnerability to their loss. We found that the mean 5' and 3' splice site scores were lower in SAV exons than HapMap exons but were not statistically significant (Table 2). Assessing exons with large numbers $(\geq 2)$ of NI-ESEs losses and/or NI-ESS gains revealed stronger 3' splice site scores in HapMap exons than SAV exons (Table 2), suggesting stronger 3' splice sites may shield some HapMap exons from the effects of ESR-changing SNPs. Nevertheless, the large overlap in splice site strengths between these two groups indicated that splice site strength could not be used to uniquely predict SAV vulnerability in exons.

\section{ESR density in exons and introns}

A major feature postulated to distinguish exons from introns is higher densities of ESEs and low or absent densities of ESSs. The exact opposite is true of introns and pseudoexons. We therefore looked at the density of exonic splicing regulators in SAV and HapMap exons using the NI-ESRs. We found that SAV exons have significantly lower densities of ESEs and higher densities of ESSs across the exon length (Table 2 and Figure 6). To confirm that these were features specific to SAV exons rather than something particular to HapMap exons, we repeated the comparison to random genome-wide exons and found very similar results, suggesting that this is a feature characteristic of SAV exons. ESR densities of SAV exons are, in many cases, more comparable to an intronic environment represented in flanking introns (mean ESE density $=0.26$, mean ESS density $=0.2$; see Materials and methods). Moreover, directly flanking SAV exons, we found that intronic sequences showed higher densities of ESSs and slightly lower densities of ESEs than around hSNP exons (Table 2). 
Table 2 Significance of exon and intron-related features for skipping SAV and HapMap exons

\begin{tabular}{|c|c|c|c|c|}
\hline Exon feature & SAV mean & hSNP sampled mean & Z-score & $P$-value \\
\hline \multicolumn{5}{|l|}{ Exon splice junction strength } \\
\hline Exon 3' SS score (all exons) & 7.811 & 8.489 & -1.90 & 0.057 \\
\hline Exon 5' SS score (all exons) & 7.885 & 8.302 & -1.43 & 0.154 \\
\hline Exon 3' SS score (with ESR) & 7.568 & 8.534 & -2.03 & 0.022 \\
\hline Exon 5' SS score (with ESR) & 8.008 & 8.371 & -1.71 & 0.230 \\
\hline \multicolumn{5}{|l|}{ Exon ESR density } \\
\hline ESEfinder density FL & 0.126 & 0.152 & -3.78 & $1.53 \times 10^{-4}$ \\
\hline NI-ESE density FL & 0.323 & 0.372 & -3.37 & $7.37 \times 10^{-4}$ \\
\hline NI-ESS density FL & 0.133 & 0.093 & 4.30 & $1.67 \times 10^{-5}$ \\
\hline ESEfinder density W40 & 0.129 & 0.153 & -3.15 & 0.0016 \\
\hline NI-ESE density W40 & 0.324 & 0.379 & -3.47 & $5.18 \times 10^{-4}$ \\
\hline $\mathrm{NI}$-ESS density W40 & 0.140 & 0.094 & 4.50 & $6.85 \times 10^{-6}$ \\
\hline \multicolumn{5}{|l|}{ Intronic ESR densities } \\
\hline Upstream NI-ESE density & 0.201 & 0.224 & -1.55 & 0.122 \\
\hline Downstream NI-ESE density & 0.235 & 0.250 & -1.06 & 0.314 \\
\hline Upstream NI-ESS density & 0.295 & 0.241 & 2.44 & 0.014 \\
\hline Downstream NI-ESS density & 0.258 & 0.210 & 2.36 & 0.018 \\
\hline
\end{tabular}

For each feature, the mean values for non-redundant SAV exons were compared to a bootstrap distribution of sampled means for HapMap exons of similar sizes (hSNP sampled mean). For exon splice junction strength, results marked 'all exons' indicate that the comparison was done using all exons in both datasets and those marked 'with ESR' indicate comparisons using only exons containing a variant with splice-associated ESR changes, that is, ESE loss and/or ESS gain. For exon ESR densities, densities were either measured across the full length of the exon (FL) or in windows of 40 bp at either side of the exon (W40). For exons <80 $\mathrm{bp}$ in length, the W40 density is the same as full length density to avoid redundancy. Intronic ESR densities were measured in the first 100 bp upstream and downstream of the exon. SS, splice site.

\section{Variants that activate de novo ectopic splice sites}

Next, we assessed features that define exonic variants that create de novo ectopic splice sites. We used a set of 54 experimentally verified examples of de novo ectopic splice site variants (Additional file 9) to discern features that distinguish our two sets of SAVs (that is, 'ectopic SAVs' and 'skipping SAVs') from each other and from hSNPs. First, to measure splice site creation, we used a simple metric, $\Delta S S$, to measure the maximum difference in splice site scores between these two sequences for all possible 5' and 3' splice sites around the variants (see Materials and methods). A large positive delta score suggests a change in the surrounding sequence towards a better scoring splice site. Requiring a relatively low $\triangle S S$ score of at least 1 captured the majority of ectopic SAVs (approximately $85 \%$ ) compared to $20 \%$ of skipping SAVs and $8 \%$ of hSNPs. We also compared the highestscoring variant-generated splice site to the natural splice site score. Over half of ectopic SAVs created ectopic splice sites that were comparable to or stronger than the natural splice site, in contrast to a tiny proportion of skipping SAVs and hSNPs (Figure 7a). Thus, these two metrics represent excellent features to discriminate ectopic SAVs from splicing SAVs. In support of this conclusion, one of the two exon skipping SAVs we predicted to also create strong ectopic splice sites, a synonymous mutation in the ATR gene, has been shown experimentally to cause a combination of both exon skipping and ectopic 5' splice site activation [37].

An additional feature of ectopic SAVs was a highly significant excess of ESS gains $\left(P=2.85 \times 10^{-15}\right)$ and ESS alterations $\left(P=1.95 \times 10^{-3}\right)$ compared to hSNPs, similar to that seen in skipping SAVs. The degree of ESS gains in ectopic SAVs was even greater than that for skipping SAVs, averaging 1.28 ESS gains per variant compared to 1.12 for skipping SAVs and 0.39 in hSNPs. When averaged across all internal constitutive exons, we found NIESS density spiked near splice junctions (Figure 7b), which was consistent with previous studies on smaller ESS datasets [15,38], suggesting a possible explanation for the excess in ESS gains. To address this further, we compared the ectopic SAVs to a set of 54 hSNPs that were tightly scored as 'ectopic-like' (but showed no evidence of splice site creation in mRNA or EST datasets; see Materials and methods). We found that ectopic SAVs had almost a 2.5-fold greater number of ESS gains (68 versus 28 ) and a 1.8 -fold greater number of ESS alterations (23 versus 13), despite both sets having similar distributions of maximum ectopic splice site scores (KS-test $P=0.11$ ). The process of creating strongly scoring splice-site consensus sequences could not, therefore, fully explain the enrichment in ESS changes in SAVs. Additional ESS creation may facilitate activation of the new ectopic splice sites by inhibiting the natural 


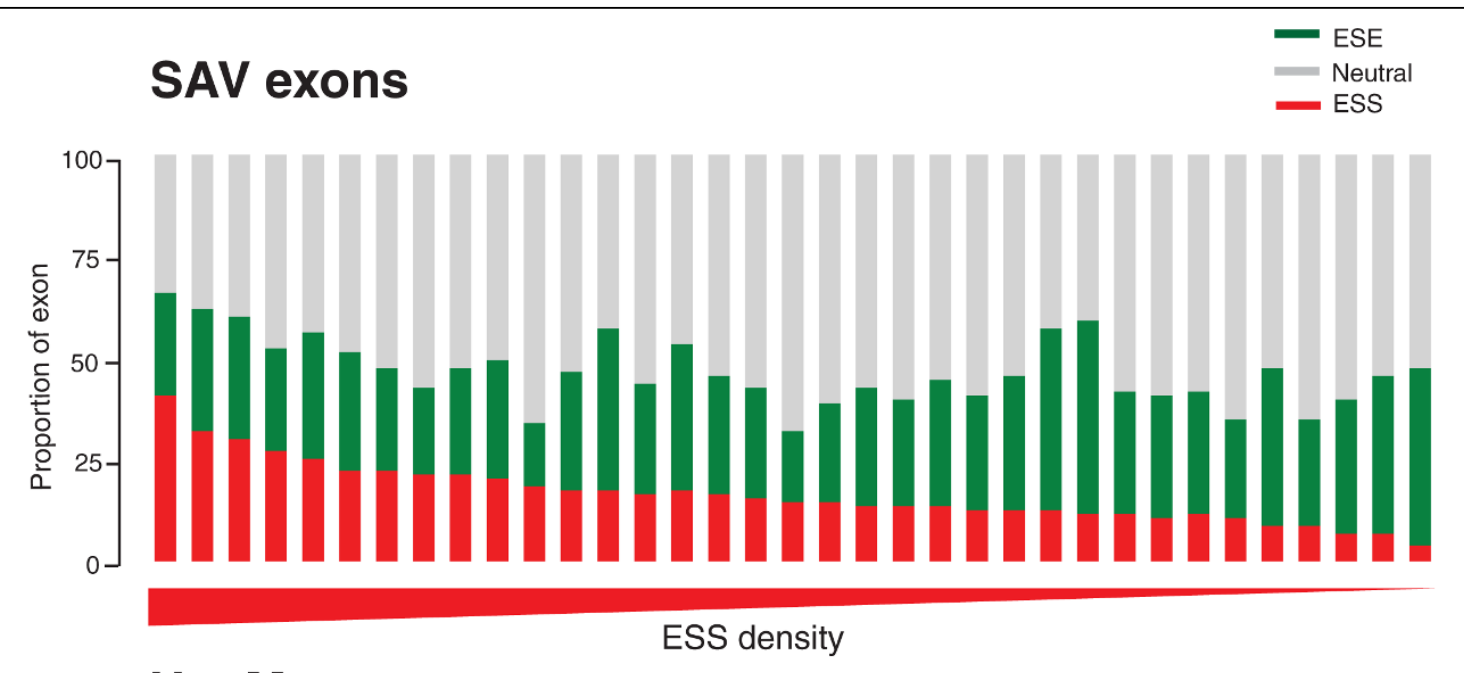

\section{HapMap exons}

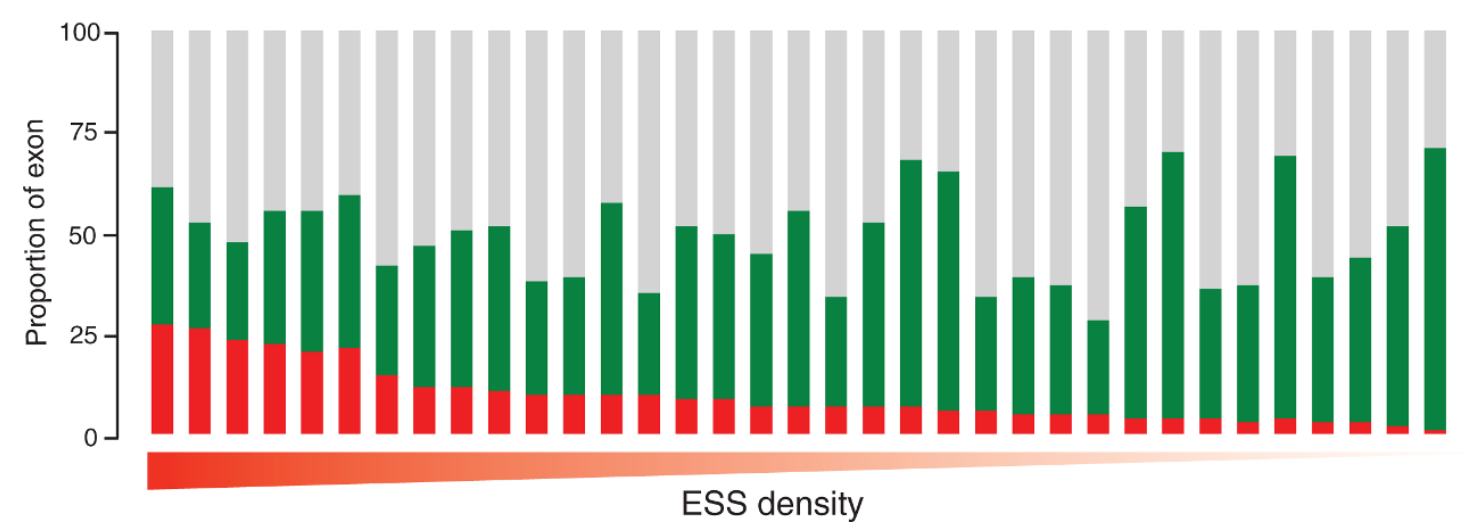

Figure 6 Exons containing SAVs have significantly lower ESE and significantly higher ESS densities than exons containing hSNPs. As an illustration, the proportion of overlapping hexamers that are considered ESEs (green), ESSs (red) or splice neutral (grey) was plotted for 35 exons containing SAVs (that cause ESE/ESS changes) and a set of 35 randomly selected, length-matched hSNP-containing exons. Exons in both sets are sorted in descending order by ESS density.

splice site. This is consistent with a functional study by Wang et al. [38] whereby ESS motifs placed between competing 5' and 3' splice sites consistently inhibited the use of the intron-proximal splice site.

Finally, the location of ectopic SAVs and 'ectopic-like' hSNPs across exons revealed very different distributions (Figure 7c). Ectopic SAVs were predominantly located in the half of the exon closest to the natural splice site they replaced. The reverse was true of 'ectopic-like' hSNPs, which were distributed across the exon in an opposite manner. These differences, in addition to the lack of silencer gains, likely account for the lack of activity of these 'ectopic-like' hSNPs.

\section{Skippy - a web tool for the detection of splice-modulating} exonic variants

It is important for researchers screening for causative variants associated with disease to have access to userfriendly bioinformatics tools that can score variants for relevant splice-associated features. In this way, variants can be either prioritized for further splicing-based functional assays or the results can be used to further elucidate the mechanism of aberrant splicing when a causal variant has been implicated. To this end, we developed a publicly accessible web-based tool, Skippy, to allow users to rapidly score human exonic variants for all relevant exon-skipping features identified in this study. As well as these features, Skippy can also be used to identify potential ectopic SAVs.

Unlike other splicing assessment tools that require laborious extraction of the exonic/intronic sequence for input and only allow a single sequence to be submitted at a time (for example, [22,39]), Skippy requires only the chromosomal location and identity of the variant alleles as input, accepting up to 200 variants at a time. Results are returned in HTML tabular form as well as a tabdelimited text file. To facilitate interpretation of results, 
(a)

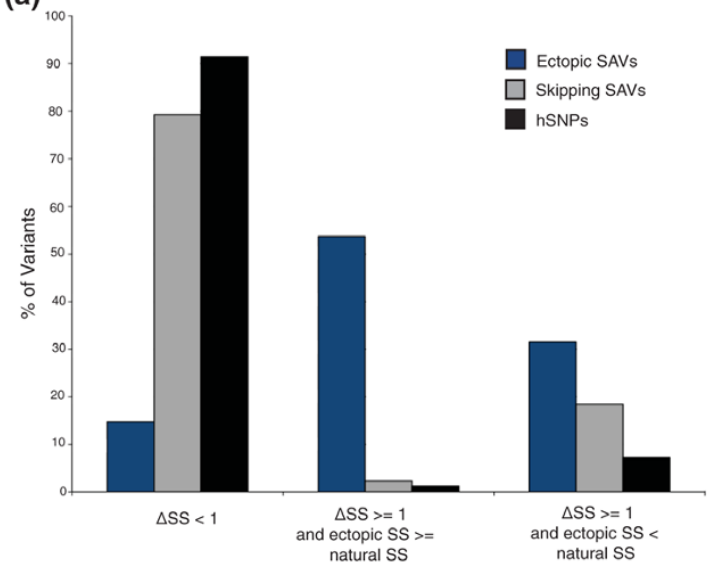

(c)

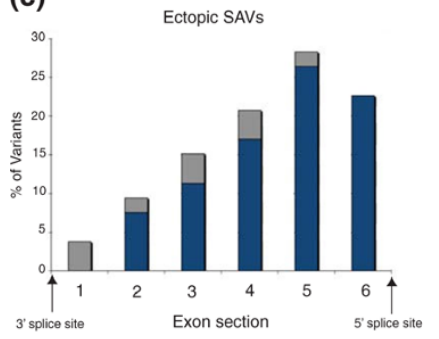

(b)

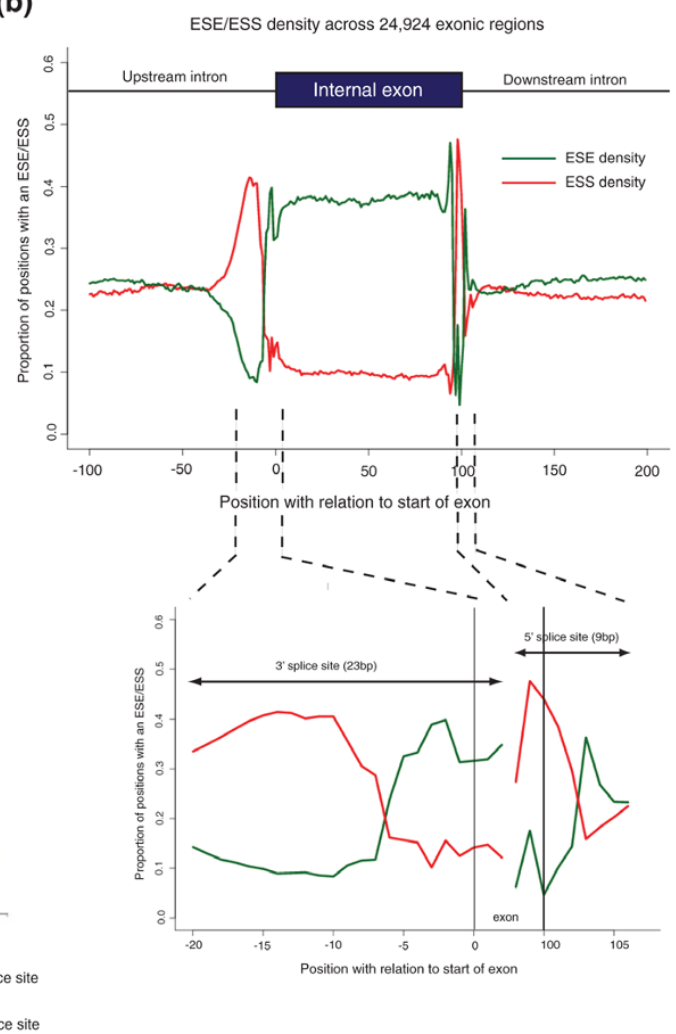

Figure 7 Features that characterize variants that activate de novo ectopic splice sites ('ectopic SAVs'). (a) Most ectopic SAVs, in contrast to hSNPS and skipping SAVs, have a large $\triangle S S$ value and create an ectopic splice site that is stronger than the natural splice site. (b) Hexamers in the vicinity of the splice junctions are largely made up of ESSs. The graph represents the proportion of positions occupied either by an ESE or ESS motif across approximately 25,000 internal exons. Each position on the graph represents the first base of a hexamer sliding across 100 bp of the upstream and downstream introns and the first and last $50 \mathrm{bp}$ of the exon. (c) Ectopic SAVs are located predominantly in the vicinity of the splice site of the same type created, that is, the majority of ectopic splice sites created are $5^{\prime}$ ectopic sites and are located towards the end of the exon close to the $5^{\prime}$ splice site. hSNPs that create a strong ectopic splice site computationally ('ectopic-like' hSNPs) are distributed across the exon in quite the opposite way, indicating the same constraints do not apply to these variants.

all scored features can be compared to distributions of hSNPs from similar genomic contexts. For example, the $\mathrm{RC}$ score for a candidate variant can be compared to a distribution of RC scores for hSNPs having similar features, such as equivalent minimum distances from the splice junctions. The web tool is freely available at [40].

\section{Discussion}

The emergence in recent years of high throughput genotyping and resequencing technologies provides an unprecedented opportunity to identify disease alleles associated with both common and rare disorders. As functional characterization is highly laborious and time consuming, computational prioritization is a preferred approach to assessing disease candidates. Exonic mutations are traditionally assessed for an effect on protein function; however, those that are translationally silent are often overlooked for roles in exon skipping and ectopic splice site creation. Moreover, variants are traditionally only considered in the vicinity of splice sites if they fall directly at splice boundaries, whereas we have shown that SAVs are enriched in regions near, but not at, the splice junctions. Any of these seemingly innocuous sequence changes may have greater consequences for gene function than a single missense mutation. We therefore showed that SAVs have novel features distinguishing them from common human polymorphisms through a succession of bioinformatics approaches and built a novel web tool for examining genomic sequence changes that are likely to affect splicing.

\section{Exon skipping SAVs cause local changes in splice regulatory elements}

Our comparative analyses identify two main types of ESR changes associated with exon skipping: the gain of sequences defined as ESSs and the loss of sequences defined as ESEs. We are the first to report that all ESS 
datasets showed a strong statistical enrichment for gain of ESSs in known SAVs with a moderate to high signal to noise ratio. Surprisingly, we also found that solely considering whether a variant causes ESE loss was a relatively weak predictor of splice-affecting events. Although the widely used ESEfinder matrices did not discriminate the known SAVs from the control groups, other ESE datasets (NI-ESE, RESCUE-ESE and Comp-ESE) showed statistically significant enrichments for ESE loss.

The study of ESR changes may not be a binary endeavor, as a single SNP can affect a number of putative overlapping binding sites. We found that SAVs are more strongly associated with the loss of large numbers of ESEs and the gain of large numbers of ESSs. This analysis also highlighted the neighborhood inference set of putative ESRs ('NI-ESRs') as providing the strongest signal for exon skipping variants. NI-ESRs are a relatively new set of predicted splice regulatory elements and have therefore been little used in clinically associated splicing studies to date. The neighborhood inference algorithm greatly enlarged the set of previously known ESEs and ESSs to cover over a third of all possible hexamers, increasing the likelihood of false positives in our ESR change analysis. We nevertheless saw impressive separation between SAVs and hSNPs, suggesting that many of these novel ESRs represent functional elements. None of our test set of known exonskipping variants was originally identified, nor confirmed using this dataset.

As an illustration, a published missense mutation in exon 12 of the $H E X B$ gene causes full exon skipping and is responsible for chronic Sandhoff's disease. The variant was identified experimentally and subsequently predicted to cause the loss of two ESEfinder sites [41]. Our analysis using NI-ESRs revealed that this mutation caused the loss of five overlapping ESEs and the creation of two overlapping ESSs (both of which were direct conversions from ESEs to ESSs). Notably, this extent of NIESR changes, unlike those for ESEfinder, scored as highly discriminative for SAVs compared to hSNPs. Furthermore, we found that concurrent loss and gain events were better predictors than single events. This fact is illustrated by the synonymous skipping mutation of exon 7 in SMN2 that destroys two overlapping ESE hexamers and creates two overlapping ESS hexamers. Functional studies of SF2/ASF and hnRNPA1 binding in this exon proved that reduced binding of SF2/ASF [11] and increased binding of hnRNPA1 [42] were responsible for reduced inclusion of the SMN2 exon.

\section{Increased silencer activity is likely for many SAVs}

Although the loss of ESEs is the most commonly assigned change in published splice-associated variant studies, increased silencer function was seen in $37 \%$ of our known exon skipping SAVs, in which each caused the gain of two or more ESSs. The clear enrichment for silencer creation in SAVs and selection against silencer acquisition in common polymorphisms suggests that this may be a major mechanism responsible for exon skipping. Furthermore, for mutations in which the mechanism of action has been experimentally studied, with the exception of $S M N 2$, none were studied for the possibility of increased silencer function. The importance of exonic silencers in splicing is further highlighted by our results showing that SAVs that cause increased exon-inclusion are likely to operate largely by the loss of ESSs. We conclude that newly created ESS sites also facilitate formation of de novo ectopic splice sites. The action of inhibiting a natural existing proximal splice site, as ESS are known to do, would be similar to those causing exon skipping when no other alternative splice site was available.

\section{Caveats}

It is important to note that despite the strong signals we identified, there are a number of limitations to solely using ESR analyses in a predictive manner. For example, even using the NI-ESR set, some SAVs were not captured with expected regulatory changes. Around 9\% of the SAVs had no relevant changes in any of the ESR datasets, indicating that putative ESRs do not cover the full spectrum of functional splicing regulatory elements or these variants act through an alternative mechanism (for example, RNA secondary structure). Furthermore, context is very important for ESR function. This fact was highlighted by a recent study of 'designer' exons that placed different combinations of known ESEs and ESSs within a minigene exon and found that exons with the same proportion of enhancers and silencers exhibited highly variable inclusion levels that were context specific according to the order of regulatory elements across the exon [43].

\section{Exon skipping SAVs occur in weakly defined exons}

Our analyses of the exonic environment suggested that an exon-skipping outcome was not necessarily solely dependant on the changes in splice regulatory elements, but may also be influenced by pre-existing features of exon definition. In this analysis SAV exons were not discernibly weaker at splice sites than other exons. However, experimental studies have indicated that weak splice sites are a factor. For example, the 5 ' splice site of SMN2 exon 7 was reported to be suboptimal through experimental and compensatory analyses [44]. This finding was not reproducible using solely computational scoring, highlighting the limitations of current in silico methods in detecting subtle but potentially significant features of exon definition. 
Along with context and strength of the splice sites, exon definition is influenced by ESE and ESS motif densities [43]. It is revealing, therefore, that SAV exons have significantly lower densities of ESEs and significantly greater densities of ESSs - a clear attribute of weak exon definition. It is currently thought that splicing efficiency increases linearly as the number of enhancer elements increases because the role of multisite splice-regulatory elements is to increase the probability of an interaction between the regulatory complex and the splicing machinery $[45,46]$. Conversely, as the number of silencer elements increases, splicing efficiency decreases [43]. Indeed, we found that ESS density of many SAV exons was more comparable to that of introns than exons. As weakly defined exons, they appear vulnerable to variants that further modulate the ESE/ESS density. Illustrating the point, some exons are vulnerable to exon skipping by numerous SAVs. Seven SAVs occur in constitutively spliced exon 12 of the CFTR gene [47,48], which has a low ESE density $(0.280$ versus 0.371 ) and exceptionally high ESS density (0.293 versus 0.091) compared to mean densities in HapMap exons.

Our results also suggest that ESS elements in the introns may play a role in the susceptibility of exons to SAVs. However, the function of ESSs in introns is not fully elucidated $[49,50]$. If ESSs in introns act mainly as intronic splicing silencers, they may make the exon increasingly reliant on exonic splicing enhancers. Such a case has been demonstrated for one of the SAVs in exon 7 of $S M N 1 / S M N 2$, where removal of a flanking intronic splicing silencer sequence compensated for the exon skipping effect [51].

\section{Conclusions}

It is becoming increasingly clear that both missense and synonymous mutations within exons can have devastating effects on gene function by modulating splicing. The location of these mutations in coding sequence, as well as the lack of a clear strategy for their identification, means that their effects are often overlooked. As a consequence, known examples are currently small in number, but are likely to be underestimated. This work provides the first large-scale analysis of exon skipping variants to computationally characterize their genomic context. We identified a number of features associated with the variants and their exonic and intronic environments that are significantly different from common splicing-neutral polymorphisms. Exon skipping SAVs are characterized by extensive loss of exonic splicing enhancers and gain of splicing silencers, often in combination. They tend to occur in regions close to splice sites and in regions under greater non-coding evolutionary selection. They also tend to occur in exons with a fairly weak environment for exon definition that is the likely cause of their vulnerability to skipping events.

Our comparative approach proved robust in identifying relevant features in other types of SAVs too. Variants that cause increased exon inclusion are characterized by ESS loss and, to a lesser degree, the gain of ESEs. Variants that activate an ectopic splice site simultaneously create large numbers of ESSs, in addition to a strong consensus splice site, and inhibit use of the natural splice site. These results provide greater insights into the possible mechanism of action of these variants and should improve strategies for identifying disease candidates. To this end, we have developed a web-based tool, Skippy, to score candidate human genomic variants for features predictive of an exon-skipping outcome or creation of an ectopic splice site.

\section{Materials and methods \\ Collation of a set of known exonic variants causing exon skipping}

In total we collated a set of 87 SAVs by extracting synonymous and missense variants from the Alternative Splicing Mutation Database [26] (with a splicing effect score $<0$ ), and from our own extensive literature searches. Only single-point variants that had been experimentally verified for exon skipping were used in the reference set. We excluded the following: nonsense variants [3] (that is, those that create a stop codon); and variants that affect the splice junction (that is, $3 \mathrm{bp}$ or less from either splice junction). Genomic positions for all 87 identified cases (32 synonymous, 55 missense) were mapped back onto the reference human genome (assembly Hg18). For the analysis of the types of ESR changes involved in increased exon inclusion, we used a set of 20 variants from the Alternative Splicing Mutation Database with splicing effect scores $>0$ ( 7 synonymous, 13 missense).

\section{Obtaining a comparator set of putatively splicing-neutral coding SNPs}

All 'phase II' HapMap SNPs (release 22), termed 'hSNPs', that were polymorphic in at least one individual were downloaded from the website [27]. SNPs had to fall within an internal coding exon (using the Ensembl known gene set, $\mathrm{v} 45.36 \mathrm{~g}$ ) and more than $3 \mathrm{bp}$ away from a splice junction. Directionality of mutations (that is, the derived alleles) utilized three-way human-chimp-macaque MulitZ alignments (hg18, panTro2, rheMac2) obtained from the UCSC Genome Browser [52] via Galaxy [53]. SNPs were retained only if there was a full three-way alignment available, chimp and macaque bases were identical, and one of the human alleles matched the ancestral chimp-macaque base. hSNPs included within the set of known SAVs were excluded from the comparator set (rs2306159, rs4647603 and rs2295682 [54], rs688 
[55] and rs17612648 [56]). In addition, four hSNPs (rs17658212, rs4963793, rs591 and rs3818562) with reported correlations to splicing changes (but unverified) [57] were also excluded. A total of 15,547 hSNPs $(7,922$ synonymous, 7,625 missense) were obtained. Derived allele frequencies of $>5 \%$ and $>10 \%$ in at least one population were assessed. We found no appreciable difference for any of our analyses when using SNPs with greater derived allele frequencies. In addition to our hSNP comparator set, we also identified a set of 80 variants from the literature that have been experimentally tested in mini-gene assays and found to have no effect on exon splicing (Additional file 2).

\section{Changes in exonic ESRs}

For our analysis, we obtained six sets of ESR sequence prediction datasets. Three comprised sets of bioinformatically defined hexamers (RESCUE-ESEs (238 ESEs) [58], NI-ESRs (979 ESEs and 496 ESSs) [19] and Ast-ESRs (285 undefined hexamers) [16]). PESX has bioinformatically defined octamers (2,096 PESEs and 974 PESSs) [18]). Fas-Hex2 contains experimentally defined ESS hexamers (176 ESSs) [15]). ESEfinder has four experimentally defined position weight matrices for SR protein binding sites [59]. Composite-ESRs are a combined set of hexamers derived from PESX, RESCUE-ESE, and FasHex2 ESS, representing 400 ESEs and 217 ESSs [60]. The effect of SNP changes on ESR predictions was calculated using a sliding window that covered all hexamers surrounding the variant. N-mers that did not 'score' as an ESE or ESS were considered splicing-neutral. Comparisons between the wild-type sequence (or ancestral allele) and the variant sequence (or derived allele) measured ESR loss (for example, an ESE to a neutral), ESR gain (for example, neutral to an ESE) and ESR alteration (for example, ESE to a different ESE). In the case of NI-ESRs, PESXs and composite ESRs, ESEs and ESSs were considered separately. For the analysis of changes in NI-ESRs, the types of changes between alleles were counted for all overlapping hexamers in which the variant was present. Expected proportions for each of the nine categories of change were calculated by permutating every base of 4,096 hexamers to all remaining bases (for example, A would be permutated to T, G and C) to give $73,728(4096 \times 3 \times 6)$ permutations. Base substitution biases were taken into account by measuring base substitutions in the hSNP derived allele set (Additional file 10) and for each permutation, weighting the ESR-change category by the proportion of base substitutions of that type.

\section{Regulatory evolutionary constraint}

An expectation-based scoring matrix measuring regulatory constraint in coding sequences was created by measuring the proportion of columns fully conserved for each of the 192 codon positions using a randomly selected set of 62,000 internal human exons in 6,428 genes from Ensembl (v47.36i). Exons were distributed genome-wide and had conserved counterparts in mouse, rat and dog genomes. Scores were assigned for each codon position by $\left(1-\operatorname{Pr}_{C O D i}\right) \times 10$ where $\operatorname{Pr}_{C O D i}$ is the proportion of columns in all the alignments that were fully conserved for codon $\mathrm{COD}$, position $i$. Scores for each codon position are therefore weighted so that they are inversely proportional to their overall conservation level. Conservation scores, measuring non-coding constraint in coding sequence, were calculated for regions surrounding variants in the hSNP and SAV sets. Orthologous sequences from human, mouse, rat and dog were extracted from 17-way MultiZ multiple alignments from the UCSC Genome Browser [52] for 5 bp either side of the SNP (representing all hexamers containing a SNP (a total of $11 \mathrm{bp}$ ) using Galaxy python scripts [53]. Smaller flanking regions were extracted if the variant was located less than $5 \mathrm{bp}$ from the splice junction. Only ungapped alignments containing at least two species in addition to human were used. The RC score surrounding a variant $\mathrm{RC}_{\mathrm{Var}}$ was calculated as follows:

$$
R C_{\text {Var }}=\frac{\sum_{i=1}^{N} s_{i} \delta_{c i}}{N}
$$

where $N$ is the number of columns in the alignment, $i$ is the column position, $S_{i}$ is the conservation status of the column ( 1 for conserved across the alignment, 0 if not fully conserved) and $\delta_{c i}$ is the weight of the score depending on the codon position of the sequence of $i$ in human (using the 192 codon scoring matrix). Pre-computed conservation scores for each base of all internal coding exons in the human genome (assembly $\mathrm{Hg} 18$ ) are available as a custom wiggle track on the UCSC genome browser from [40]. For all statistical analyses, only variants with non-overlapping regions were used to avoid bias. To compare conservation in SAV exons and HapMap exons, human/mouse/rat/dog multiple alignments were extracted across all exons represented in both sets. We computed the proportion of non-synonymous sites and proportion of columns that were fully conserved across the alignment within each exon.

\section{Exon-based features}

All exons (and their flanking intronic sequences) containing SAVs and hSNPs were extracted from the human genome (assembly Hg18) using the Ensembl API [61] always using the largest exon isoform (except in the case of intron retention events). A genome-wide 
set of internal human cassette exons was downloaded from the Hollywood exon annotation database [36]. We retained exons between 20 and 1,000 bp with canonical GT-AG splice junctions, solely annotated as constitutive or alternatively spliced, obtaining 105,932 exons. Of these, 98,692 were annotated as constitutive and 7,240 were alternatively spliced. A simulated distribution of expected exon lengths for hSNPs, given a random distribution across the genome of 1 every thousand bases [27], was calculated for each exon length $n$ (going from $20 \mathrm{bp}$ to $1,000 \mathrm{bp}$ ) using the formula $f r(n)=p S N P \times o b s(n)$ where $p S N P=0.001$ and $o b s(n)$ is the observed number of exons for length $n$ in the set of Hollywood exons. Splice site strength at both the 5' and 3' splice junctions was measured using the MaxEntScan maximum entropy scoring program [62] with default settings. We calculated ESR density within an exon by scanning a window of size $n$ (depending on size of the ESR) across the length of the exon, and then dividing by the number of windows that scored as an ESE or ESS by the total number of windows. ESEfinder densities were calculated differently due to their encoding as position weight matrices of differing length. The density of each of the four position weight matrices within the exon was calculated separately using the windowing method and summed to make an overall density. We excluded the possibility of ascertainment bias for exon features due to expression levels by comparing 68 exons from SAVcontaining genes that contained hSNPs but not SAVs to the rest of the hSNP exon dataset. We found no significant differences for ESR change or exon characteristics (such as exon length, splice site strength, ESE/ ESS density, and so on) compared to other hSNPs or their exons.

\section{Intron-based features}

All ESE/ESS densities of intronic sequences were measured using the NI-ESR set in the same way as for exons, on $100 \mathrm{bp}$ of sequence directly flanking each side of the exon (excluding the conserved GT-AG splice site dinucleotides). Any exons with a flanking intron of less than $102 \mathrm{bp}$ were excluded.

\section{Variants that activate de novo cryptic splice sites}

From the DBASS3 [63] and DBASS5 [64] databases, we obtained 54 experimentally verified examples of exonic mutations that activate a de novo (that is, not pre-existing) ectopic 5' or 3' splice site and are located more than 3 bp away from either splice junction and mapped them back on to the human genome assembly hg18 (Additional file 9). We measured potential creation of de novo splice sites by a variant using a metric $\Delta S S$. $\Delta S S$ represents the maximum change in values for either 5 , or 3' MaxEnt splice site scores between variant and wild type, that is, $\Delta S S=\max \left(\Delta 5^{\prime} S S \mid \Delta 3^{\prime} S S\right) . \Delta 5^{\prime} S S=\left(M E_{\text {var }}\right.$ $\left.M E_{w t}\right)$ where $M E_{v a r}$ and $M E_{w t}$ are the $5^{\prime}$ MaxEntScan scores for the sequence including the variant and wildtype allele, respectively. Similarly $\triangle 3$ 'SS is calculated in the same way but using the 3' MaxEntScan scoring program. $\triangle 5^{\prime} S S$ and $\triangle 3^{\prime} S S$ were calculated for every appropriate sequence window ( $9 \mathrm{bp}$ for $5^{\prime}$ splice sites and 23 bp for 3' splice sites) in which a variant could play a role, sliding the window 1 bp each time. A comparator set of the top 54 ectopic-like hSNPs were created by choosing those hSNPs with the greatest scores for putative ectopic splice sites created by the variant, a $\Delta S S \geq 1$ and no evidence of ectopic splice site creation as judged by mRNA and EST evidence from GenBank. Interestingly, prior to using the last filter, we found two of the top 56 hSNPs have strong evidence of causing ectopic splice site creation (rs7529443 (G->A) and rs2863095 $(\mathrm{G}->\mathrm{A})$ ). This strategy, used with other evidence, can be used to identify novel ectopic splice site creating SNPs. To identify whether natural splice sites are predominantly made up from sequences defined as ESSs, we used DNA sequence from $100 \mathrm{bp}$ within the exon (the first and last $50 \mathrm{bp}$ in cases where the exon length $>100$ $\mathrm{bp}$ ) in addition to $100 \mathrm{bp}$ from the flanking upstream and downstream introns from a subset of the constitutively spliced exons with canonical GT-AG splice junctions from the Hollywood database. We therefore required that exons be at least $100 \mathrm{bp}$ in length and contain flanking introns of at least $200 \mathrm{bp}$ in length (so as not to contain mixed signal from nearby exons), leaving 24,924 exons.

\section{Statistical analysis}

Unless otherwise indicated, we carried out a bootstrap analysis to compare SAVs against the hSNP set by randomly sampling sets of the same size and proportion of synonymous and non-synonymous as the SAVs without replacement (using the Perl module Math:: Random) from the hSNPs $1 \times 10^{5}$ times. For the analysis involving ectopic SAVs, only hSNPs with a $\Delta S S$ score of 0 were compared. The number of cases sampled from the hSNP set for bootstrap analysis depended on whether the parameter was variant-based (that is, dependant on the variant, such as changes in ESRs) or exon-based. For variant-based parameters, all variants were used. As some SAVs or hSNPs fall within the same exon, exon-based parameters utilized only unique exons within the set to avoid biasing the analysis. Z-scores were calculated as long as the distribution of sampled values passed the Shapiro-Wilk test for normality $(P>0.05)$ otherwise the lowest empirical $P$-value was presented. $P$-values were derived from $\mathrm{Z}$ scores calculated using: 


$$
z=\frac{x-\mu}{\sigma}
$$

where $x$ is the feature value (or mean value) for SAVs, $\mu$ is the mean and $\sigma$ is the standard deviation of a distribution of feature values (or mean values) of randomly sampled hSNPs. A more stringent $\alpha$ value of 0.01 was used to determine statistical significance given the large number of statistical comparisons carried out. Comparison of the proportion of SNPs showing changes in different motif positions within ESEfinder motifs for SAVs and hSNPs was carried out using $\chi^{2}$ with Yates correction. Exon length distributions were compared using both the sampling approach above, as well as the K-S test as implemented in the $\mathrm{R}$ statistics package.

Additional file 1: Table S1 List of 87 synonymous and missense spliceaffecting genome variants (SAVs) that cause exon skipping used for analysis in this study. The variants are derived from

$[12,13,37,41,44,47,48,54-56,65-103]$.

Click here for file

[http://www.biomedcentral.com/content/supplementary/gb-2010-11-2r20-S1.pdf]

Additional file 2: Table S4 List of 80 synonymous and missense variants that have been experimentally tested in mini-gene constructs and do not cause changes in splicing. The variants are derived from [74104105106].

Click here for file

[http://www.biomedcentral.com/content/supplementary/gb-2010-11-2r20-S2.pdf]

Additional file 3: Table S5 (a) Full results of ESR changes and bootstrap analysis of exon skipping SAVs vs. hSNPs, (b) splice-neutral variants vs. hSNPs, (c) SAVs that cause exon inclusion vs. hSNPs, (d) ectopic SAVs vs. hSNPs and (e) ectopic-like hSNPs vs. hSNPs with a $\triangle$ SS of 0.

Click here for file

[http://www.biomedcentral.com/content/supplementary/gb-2010-11-2r20-S3.pdf]

Additional file 4: Figure S2. Proportion of exon skipping SAVs and hSNPs that destroy an ESEfinder motif and the position in which they occur across four binding sites Set of graphs illustrating that exon-skipping SAVs are significantly overrepresented within certain positions across the four ESEfinder matrices.

Click here for file

[http://www.biomedcentral.com/content/supplementary/gb-2010-11-2r20-S4.pdf ]

Additional file 5: Methods and Results S1. Local RNA secondary structure analysis Methods and results for an analysis on whether using local RNA secondary structure as a filter improves our ability to distinguish exon skipping SAVs from hSNPs. Our results suggest that using this filter does not improve our ability to predict SAVs although a small number of SAVs may arise from the indirect uncovering of ESS motifs by changes in local RNA secondary structure.

Click here for file

[http://www.biomedcentral.com/content/supplementary/gb-2010-11-2r20-S5.pdf ]

Additional file 6: Table S2 List of 20 variants that cause increased exon inclusion. The variants are derived from [4447104107108].

Click here for file

[http://www.biomedcentral.com/content/supplementary/gb-2010-11-2r20-S6.pdf]
Additional file 7: Figure S3. The RC score is influenced by distance from the splice junction but not by exon length Two plots that show that mean RC score is negatively correlated with minimum distance from a splice junction (top) but not correlated with exon length (bottom). Click here for file

[http://www.biomedcentral.com/content/supplementary/gb-2010-11-2r20-S7.pdf ]

Additional file 8: Figure S4. Distribution of exon lengths for SAV exons versus HapMap and genome-wide exons Only distributions of exon lengths up to 600 bp were plotted for clarity. Genome-wide exons were divided into constitutively spliced (CE) and alternatively spliced (AS) as defined by the Hollywood database [36]. A fifth, expected set of exons represents a set of exon lengths we would expect given the average distribution of hSNPs across the genome and fits the real distribution of HapMap exons closely.

Click here for file

[http://www.biomedcentral.com/content/supplementary/gb-2010-11-2r20-S8.pdf ]

Additional file 9: Table S3 List of 54 variants that cause de novo $5^{\prime}$ or $3^{\prime}$ ectopic splice site activation. The variants are derived from $[4,37,86,109-$ 154].

Click here for file

[http://www.biomedcentral.com/content/supplementary/gb-2010-11-2r20-S9.pdf]

Additional file 10: Figure S1. Distribution of nucleotide substitutions types in 15,547 HapMap derived alleles ('hSNPs'), 87 exon skipping SAVs ('skipping SAVs') and 54 SAVs that create a de novo ectopic splice site ('ectopic SAVs') Base substitution distributions in hSNPs were used a background nucleotide substitution rates in calculating an expected distribution of ESE/ESS changes of the NI-ESR hexamer set (Figure 3). Significant differences in distributions between skipping SAVs and hSNPs (such as that seen in $\mathrm{A}->\mathrm{T}, \mathrm{T}->\mathrm{C}$ and $\mathrm{G}->\mathrm{T}$ ) and ectopic SAVs (A->T, A->C, T->A, C->G) while potentially of biological interest, should be treated with caution due to small number of skipping and ectopic SAVs and large discrepancy in dataset size between these sets and HapMap SNPs.

Click here for file

[ http://www.biomedcentral.com/content/supplementary/gb-2010-11-2r20-S10.pdf]

\section{Abbreviations}

bp: base pair; ESE: exonic splicing enhancer; ESR: exonic splicing regulatory sequence; ESS: exonic splicing silencer; EST: expressed sequence tag; hnRNP: heterogeneous nuclear ribonucleoprotein; hSNP: HapMap single nucleotide polymorphism; K-S: Kolmogorov-Smirnov; NI: neighborhood inference; RC: regulatory constraint; SAV: splice-affecting genome variant; SNP: single nucleotide polymorphism; SR: serine/arginine rich.

\section{Acknowledgements}

We thank Giovanni Parmigiani for statistical advice, Michael Hiller for help on calculating RNA structure probability of unpaired values and Dirk Holste for help on extracting data from the Hollywood database. Thanks must also go to Tyra Wolfsberg, Chris Pan, Mark Fredriksen and Gongwei Jiang for help setting up the Skippy database. We would also like to thank Lawrence Brody and the NIH Fellows Editorial Board for advice and critical reading of the manuscript. This work was supported by the Intramural Program of the National Human Genome Research Institute.

\section{Author details}

${ }^{1}$ Genomic Functional Analysis Section, National Human Genome Research Institute, National Institutes of Health, Rockville, Maryland 20892, USA. ${ }^{2}$ Comparative Genomics Unit, National Human Genome Research Institute, National Institutes of Health, Rockville, Maryland 20892, USA. 


\section{Authors' contributions}

AW collated all splice variant datasets, designed and performed the experiments, analyzed the data, coded and implemented the Skippy program/webtool and drafted the manuscript. JCM contributed the hSNP variant dataset and conceived of the SNP density/exon length analysis. LE conceived of the study, and participated in its design and coordination and helped write the manuscript. All authors read and approved the final manuscript.

Received: 16 December 2009 Revised: 3 February 2010 Accepted: 16 February 2010 Published: 16 February 2010

\section{References}

1. Ingram EM, Spillantini MG: Tau gene mutations: dissecting the pathogenesis of FTDP-17. Trends Mol Med 2002, 8:555-562.

2. Krawczak M, Reiss J, Cooper DN: The mutational spectrum of single basepair substitutions in mRNA splice junctions of human genes: causes and consequences. Hum Genet 1992, 90:41-54.

3. Cartegni L, Chew SL, Krainer AR: Listening to silence and understanding nonsense: exonic mutations that affect splicing. Nat Rev Genet 2002, 3:285-298.

4. Eriksson M, Brown WT, Gordon LB, Glynn MW, Singer J, Scott L, Erdos MR, Robbins CM, Moses TY, Berglund P, Dutra A, Pak E, Durkin S, Csoka AB, Boehnke M, Glover TW, Collins FS: Recurrent de novo point mutations in lamin A cause Hutchinson-Gilford progeria syndrome. Nature 2003, 423:293-298.

5. Venables JP: Downstream intronic splicing enhancers. FEBS Lett 2007, 581:4127-4131

6. Murray Jl, Voelker RB, Henscheid KL, Warf MB, Berglund JA: Identification of motifs that function in the splicing of non-canonical introns. Genome Biol 2008, 9:R97.

7. Jurica MS, Moore MJ: Pre-mRNA splicing: awash in a sea of proteins. Mol Cell 2003, 12:5-14

8. Zuo P, Maniatis T: The splicing factor U2AF35 mediates critical proteinprotein interactions in constitutive and enhancer-dependent splicing. Genes Dev 1996, 10:1356-1368.

9. Kan JL, Green MR: Pre-mRNA splicing of IgM exons M1 and M2 is directed by a juxtaposed splicing enhancer and inhibitor. Genes Dev 1999, 13:462-471.

10. Liu HX, Cartegni L, Zhang MQ, Krainer AR: A mechanism for exon skipping caused by nonsense or missense mutations in BRCA1 and other genes. Nat Genet 2001, 27:55-58

11. Cartegni L, Krainer AR: Disruption of an SF2/ASF-dependent exonic splicing enhancer in SMN2 causes spinal muscular atrophy in the absence of SMN1. Nat Genet 2002, 30:377-384.

12. Boichard A, Venet $L$, Naas T, Boutron A, Chevret L, de Baulny HO, De Lonlay P, Legrand A, Nordman P, Brivet M: Two silent substitutions in the PDHA1 gene cause exon 5 skipping by disruption of a putative exonic splicing enhancer. Mol Genet Metab 2008, 93:323-330.

13. Moseley $C T$, Mullis PE, Prince MA, Phillips JA: An exon splice enhancer mutation causes autosomal dominant GH deficiency. J Clin Endocrinol Metab 2002, 87:847-852.

14. Mayeda A, Screaton GR, Chandler SD, Fu XD, Krainer AR: Substrate specificities of SR proteins in constitutive splicing are determined by their RNA recognition motifs and composite pre-mRNA exonic elements. Mol Cell Biol 1999, 19:1853-1863.

15. Wang Z, Rolish ME, Yeo G, Tung V, Mawson M, Burge CB: Systematic identification and analysis of exonic splicing silencers. Cell 2004 119:831-845.

16. Goren A, Ram O, Amit M, Keren H, Lev-Maor G, Vig I, Pupko T, Ast G: Comparative analysis identifies exonic splicing regulatory sequences the complex definition of enhancers and silencers. Mol Cell 2006, 22:769-781.

17. Fairbrother WG, Yeh RF, Sharp PA, Burge CB: Predictive identification of exonic splicing enhancers in human genes. Science 2002, 297:1007-1013.

18. Zhang $\mathrm{XH}$, Chasin LA: Computational definition of sequence motifs governing constitutive exon splicing. Genes Dev 2004, 18:1241-1250

19. Stadler MB, Shomron N, Yeo GW, Schneider A, Xiao X, Burge CB: Inference of splicing regulatory activities by sequence neighborhood analysis. PLOS Genet 2006, 2:e191.
20. Hartmann L, Theiss S, Niederacher D, Schaal H: Diagnostics of pathogenic splicing mutations: does bioinformatics cover all bases?. Front Biosci 2008, 13.3252-3272

21. Buratti $E$, Baralle FE: Influence of RNA secondary structure on the premRNA splicing process. Mol Cell Biol 2004, 24:10505-10514.

22. Hiller M, Zhang Z, Backofen R, Stamm S: Pre-mRNA secondary structures influence exon recognition. PLoS Genet 2007, 3:e204.

23. Andersen $M C$, Engstrom $P G$, Lithwick $S$, Arenillas $D$, Eriksson $P$, Lenhard $B$, Wasserman WW, Odeberg J: In silico detection of sequence variations modifying transcriptional regulation. PLOS Comput Biol 2008, 4:e5.

24. Drake JA, Bird C, Nemesh J, Thomas DJ, Newton-Cheh C, Reymond A, Excoffier L, Attar H, Antonarakis SE, Dermitzakis ET, Hirschhorn JN: Conserved noncoding sequences are selectively constrained and not mutation cold spots. Nat Genet 2006, 38:223-227.

25. Fairbrother WG, Holste D, Burge CB, Sharp PA: Single nucleotide polymorphism-based validation of exonic splicing enhancers. PLoS Biol 2004, 2:E268.

26. Bechtel JM, Rajesh P, llikchyan I, Deng Y, Mishra PK, Wang Q, Wu X Afonin KA, Grose WE, Wang Y, Khuder S, Fedorov A: The Alternative Splicing Mutation Database: a hub for investigations of alternative splicing using mutational evidence. BMC Res Notes 2008, 1:3

27. Frazer KA, Ballinger DG, Cox DR, Hinds DA, Stuve LL, Gibbs RA, Belmont JW, Boudreau A, Hardenbol P, Leal SM, Pasternak S, Wheeler DA, Willis TD, Yu F, Yang H: A second generation human haplotype map of over 3.1 million SNPs. Nature 2007, 449:851-861.

28. Xu R, Teng J, Cooper TA: The cardiac troponin T alternative exon contains a novel purine-rich positive splicing element. Mol Cell Biol 1993, 13:3660-3674.

29. Parmley JL, Urrutia AO, Potrzebowski L, Kaessmann H, Hurst LD: Splicing and the evolution of proteins in mammals. PLOS Biol 2007, 5:e14.

30. Parmley JL, Hurst LD: Exonic splicing regulatory elements skew synonymous codon usage near intron-exon boundaries in mammals. Mol Biol Evol 2007, 24:1600-1603.

31. Yeo G, Hoon S, Venkatesh B, Burge CB: Variation in sequence and organization of splicing regulatory elements in vertebrate genes. Proc Natl Acad Sci USA 2004, 101:15700-15705.

32. Sorek R, Ast G: Intronic sequences flanking alternatively spliced exons are conserved between human and mouse. Genome Res 2003, 13:1631-1637.

33. Parmley JL, Chamary JV, Hurst LD: Evidence for purifying selection against synonymous mutations in mammalian exonic splicing enhancers. Mol Biol Evol 2006, 23:301-309.

34. Lareau LF, Inada M, Green RE, Wengrod JC, Brenner SE: Unproductive splicing of SR genes associated with highly conserved and ultraconserved DNA elements. Nature 2007, 446:926-929.

35. Waterston RH, Lindblad-Toh K, Birney E, Rogers J, Abril JF, Agarwal P, Agarwala R, Ainscough R, Alexandersson M, An P, Antonarakis SE, Attwood J, Baertsch R, Bailey J, Barlow K, Beck S, Berry E, Birren B, Bloom T, Bork P, Botcherby M, Bray N, Brent MR, Brown DG, Brown SD, Bult C, Burton J, Butler J, Campbell RD, Carninci $P$, et al: Initial sequencing and comparative analysis of the mouse genome. Nature 2002, 420:520-562

36. Holste $D$, Huo G, Tung V, Burge CB: HOLLYWOOD: a comparative relational database of alternative splicing. Nucleic Acids Res 2006, 34 D56-62.

37. O'Driscoll M, Ruiz-Perez VL, Woods CG, Jeggo PA, Goodship JA: A splicing mutation affecting expression of ataxia-telangiectasia and Rad3-related protein (ATR) results in Seckel syndrome. Nat Genet 2003, 33:497-501.

38. Wang Z, Xiao X, Van Nostrand E, Burge CB: General and specific functions of exonic splicing silencers in splicing control. Mol Cell 2006, 23:61-70.

39. Schwartz S, Hall E, Ast G: SROOGLE: webserver for integrative, userfriendly visualization of splicing signals. Nucleic Acids Res 2009, 37 : W189-192.

40. SKIPPY. http://research.nhgri.nih.gov/skippy/.

41. Santoro M, Modoni A, Sabatelli M, Madia F, Piemonte F, Tozzi G, Ricci E, Tonali PA, Silvestri G: Chronic GM2 gangliosidosis type Sandhoff associated with a novel missense HEXB gene mutation causing a double pathogenic effect. Mol Genet Metab 2007, 91:111-114.

42. Kashima T, Manley JL: A negative element in SMN2 exon 7 inhibits splicing in spinal muscular atrophy. Nat Genet 2003, 34:460-463.

43. Zhang XH, Arias MA, Ke S, Chasin LA: Splicing of designer exons reveals unexpected complexity in pre-mRNA splicing. Rna 2009, 15:367-376. 
44. Singh NN, Androphy EJ, Singh RN: In vivo selection reveals combinatorial controls that define a critical exon in the spinal muscular atrophy genes. Rna 2004, 10:1291-1305.

45. Hertel KJ, Maniatis T: The function of multisite splicing enhancers. Mol Cell 1998, 1:449-455.

46. Graveley BR, Hertel K, Maniatis T: A systematic analysis of the factors that determine the strength of pre-mRNA splicing enhancers. EMBO J 1998, 17:6747-6756.

47. Pagani F, Raponi M, Baralle FE: Synonymous mutations in CFTR exon 12 affect splicing and are not neutral in evolution. Proc Natl Acad Sci USA 2005, 102:6368-6372

48. Tzetis M, Efthymiadou A, Strofalis S, Psychou P, Dimakou A, Pouliou E, Doudounakis $S$, Kanavakis E: CFTR gene mutations - including three novel nucleotide substitutions - and haplotype background in patients with asthma, disseminated bronchiectasis and chronic obstructive pulmonary disease. Hum Genet 2001, 108:216-221.

49. Yeo GW, Nostrand EL, Liang TY: Discovery and analysis of evolutionarily conserved intronic splicing regulatory elements. PLoS Genet 2007, 3:e85.

50. Wang Z, Burge CB: Splicing regulation: from a parts list of regulatory elements to an integrated splicing code. Rna 2008, 14:802-813.

51. Singh NK, Singh NN, Androphy EJ, Singh RN: Splicing of a critical exon of human Survival Motor Neuron is regulated by a unique silencer element located in the last intron. Mol Cell Biol 2006, 26:1333-1346.

52. Kuhn RM, Karolchik D, Zweig AS, Trumbower H, Thomas DJ, Thakkapallayil A, Sugnet CW, Stanke M, Smith KE, Siepel A, Rosenbloom KR, Rhead B, Raney BJ, Pohl A, Pedersen JS, Hsu F, Hinrichs AS, Harte RA, Diekhans M, Clawson H, Bejerano G, Barber GP, Baertsch R, Haussler D, Kent WJ: The UCSC genome browser database: update 2007. Nucleic Acids Res 2007, 35:D668-673.

53. Giardine B, Riemer C, Hardison RC, Burhans R, Elnitski L, Shah P, Zhang Y, Blankenberg D, Albert I, Taylor J, Miller W, Kent WJ, Nekrutenko A: Galaxy: a platform for interactive large-scale genome analysis. Genome Res 2005, 15:1451-1455.

54. Hull J, Campino S, Rowlands K, Chan MS, Copley RR, Taylor MS, Rockett K, Elvidge $G$, Keating B, Knight J, Kwiatkowski D: Identification of common genetic variation that modulates alternative splicing. PLoS Genet 2007, 3 e99.

55. Zhu H, Tucker HM, Grear KE, Simpson JF, Manning AK, Cupples LA, Estus S: A common polymorphism decreases low-density lipoprotein receptor exon 12 splicing efficiency and associates with increased cholesterol. Hum Mol Genet 2007, 16:1765-1772.

56. Zilch CF, Walker AM, Timon M, Goff LK, Wallace DL, Beverley PC: A point mutation within CD45 exon $A$ is the cause of variant CD45RA splicing in humans. Eur I Immunol 1998, 28:22-29.

57. Kwan T, Benovoy D, Dias C, Gurd S, Provencher C, Beaulieu P, Hudson TJ, Sladek R, Majewski J: Genome-wide analysis of transcript isoform variation in humans. Nat Genet 2008, 40:225-231.

58. Fairbrother WG, Yeo GW, Yeh R, Goldstein P, Mawson M, Sharp PA, Burge CB: RESCUE-ESE identifies candidate exonic splicing enhancers in vertebrate exons. Nucleic Acids Res 2004, 32:W187-190.

59. Cartegni L, Wang J, Zhu Z, Zhang MQ, Krainer AR: ESEfinder: A web resource to identify exonic splicing enhancers. Nucleic Acids Res 2003, 31:3568-3571.

60. Ke S, Zhang $X H$, Chasin LA: Positive selection acting on splicing motifs reflects compensatory evolution. Genome Res 2008, 18:533-543.

61. Flicek P, Aken BL, Beal K, Ballester B, Caccamo M, Chen Y, Clarke L, Coates G, Cunningham F, Cutts T, Down T, Dyer SC, Eyre T, Fitzgerald S, Fernandez-Banet J, Graf S, Haider S, Hammond M, Holland R, Howe KL, Howe K, Johnson N, Jenkinson A, Kahari A, Keefe D, Kokocinski F, Kulesha E, Lawson D, Longden I, Megy K, et al: Ensembl 2008. Nucleic Acids Res 2008, 36:D707-714.

62. Yeo G, Burge CB: Maximum entropy modeling of short sequence motifs with applications to RNA splicing signals. I Comput Biol 2004, 11:377-394

63. Vorechovsky I: Aberrant 3' splice sites in human disease genes: mutation pattern, nucleotide structure and comparison of computational tools that predict their utilization. Nucleic Acids Res 2006, 34:4630-4641.

64. Buratti E, Chivers M, Kralovicova J, Romano M, Baralle M, Krainer AR, Vorechovsky I: Aberrant 5' splice sites in human disease genes: mutation pattern, nucleotide structure and comparison of computational tools that predict their utilization. Nucleic Acids Res 2007, 35:4250-4263.
65. Eeds AM, Mortlock D, Wade-Martins R, Summar ML: Assessing the functional characteristics of synonymous and nonsynonymous mutation candidates by use of large DNA constructs. Am J Hum Genet 2007, 80:740-750.

66. McVety S, Li L, Gordon PH, Chong G, Foulkes WD: Disruption of an exon splicing enhancer in exon 3 of MLH1 is the cause of HNPCC in a Quebec family. J Med Genet 2006, 43:153-156.

67. Lorson $\mathrm{CL}$, Hahnen $\mathrm{E}$, Androphy $\mathrm{EJ}$, Wirth B: A single nucleotide in the SMN gene regulates splicing and is responsible for spinal muscular atrophy. Proc Natl Acad Sci USA 1999, 96:6307-6311.

68. Montera M, Piaggio F, Marchese C, Gismondi V, Stella A, Resta N, Varesco L, Guanti G, Mareni C: A silent mutation in exon 14 of the APC gene is associated with exon skipping in a FAP family. J Med Genet 2001, 38:863-867.

69. Auricchio A, Griseri P, Carpentieri ML, Betsos N, Staiano A, Tozzi A, Priolo M, Thompson H, Bocciardi R, Romeo G, Ballabio A, Ceccherini I: Double heterozygosity for a RET substitution interfering with splicing and an EDNRB missense mutation in Hirschsprung disease. Am J Hum Genet 1999, 64:1216-1221.

70. Llewellyn DH, Scobie GA, Urquhart AJ, Whatley SD, Roberts AG, Harrison PR, Elder GH: Acute intermittent porphyria caused by defective splicing of porphobilinogen deaminase RNA: a synonymous codon mutation at -22 bp from the 5' splice site causes skipping of exon 3. J Med Genet 1996, 33:437-438.

71. Liu W, Qian C, Francke U: Silent mutation induces exon skipping of fibrillin-1 gene in Marfan syndrome. Nat Genet 1997, 16:328-329.

72. Ploos van Amstel JK, Bergman AJ, van Beurden EA, Roijers JF, Peelen $T$, Berg van den IE, Poll-The BT, Kvittingen EA, Berger R: Hereditary tyrosinemia type 1: novel missense, nonsense and splice consensus mutations in the human fumarylacetoacetate hydrolase gene; variability of the genotype-phenotype relationship. Hum Genet 1996, 97:51-59.

73. Colapietro P, Gervasini C, Natacci F, Rossi L, Riva P, Larizza L: NF1 exon 7 skipping and sequence alterations in exonic splice enhancers (ESEs) in a neurofibromatosis 1 patient. Hum Genet 2003, 113:551-554.

74. Baralle M, Skoko N, Knezevich A, De Conti L, Motti D, Bhuvanagiri M, Baralle D, Buratti E, Baralle FE: NF1 mRNA biogenesis: effect of the genomic milieu in splicing regulation of the NF1 exon 37 region. FEBS Lett 2006, 580:4449-4456

75. Ferrari S, Giliani S, Insalaco A, Al-Ghonaium A, Soresina AR, Loubser M, Avanzini MA, Marconi M, Badolato R, Ugazio AG, Levy Y, Catalan N, Durandy A, Tbakhi A, Notarangelo LD, Plebani A: Mutations of CD40 gene cause an autosomal recessive form of immunodeficiency with hyper IgM. Proc Natl Acad Sci USA 2001, 98:12614-12619.

76. Cardozo AK, De Meirleir L, Liebaers I, Lissens W: Analysis of exonic mutations leading to exon skipping in patients with pyruvate dehydrogenase E1 alpha deficiency. Pediatr Res 2000, 48:748-753.

77. Tran VK, Takeshima Y, Zhang Z, Yagi M, Nishiyama A, Habara Y, Matsuo M: Splicing analysis disclosed a determinant single nucleotide for exon skipping caused by a novel intraexonic four-nucleotide deletion in the dystrophin gene. J Med Genet 2006, 43:924-930.

78. Ramser J, Abidi FE, Burckle CA, Lenski C, Toriello H, Wen G, Lubs HA, Engert S, Stevenson RE, Meindl A, Schwartz CE, Nguyen G: A unique exonic splice enhancer mutation in a family with X-linked mental retardation and epilepsy points to a novel role of the renin receptor. Hum Mol Genet 2005, 14:1019-1027.

79. Lenski C, Kooy RF, Reyniers E, Loessner D, Wanders RJ, Winnepenninckx B, Hellebrand H, Engert S, Schwartz CE, Meindl A, Ramser J: The reduced expression of the $\mathrm{HADH} 2$ protein causes $\mathrm{X}$-linked mental retardation, choreoathetosis, and abnormal behavior. Am J Hum Genet 2007, 80:372-377.

80. Steingrimsdottir H, Rowley G, Dorado G, Cole J, Lehmann AR: Mutations which alter splicing in the human hypoxanthine-guanine phosphoribosyltransferase gene. Nucleic Acids Res 1992, 20:1201-1208.

81. Nielsen KB, Sorensen S, Cartegni L, Corydon TJ, Doktor TK, Schroeder LD, Reinert LS, Elpeleg O, Krainer AR, Gregersen N, Kjems J, Andresen BS: Seemingly neutral polymorphic variants may confer immunity to splicing-inactivating mutations: a synonymous SNP in exon 5 of MCAD protects from deleterious mutations in a flanking exonic splicing enhancer. Am J Hum Genet 2007, 80:416-432. 
82. Gromoll J, Lahrmann L, Godmann M, Muller T, Michel C, Stamm S, Simoni M: Genomic checkpoints for exon 10 usage in the luteinizing hormone receptor type 1 and type 2. Mol Endocrinol 2007, 21:1984-1996.

83. Nystrom-Lahti M, Holmberg M, Fidalgo P, Salovaara R, de la Chapelle A, Jiricny J, Peltomaki P: Missense and nonsense mutations in codon 659 of MLH1 cause aberrant splicing of messenger RNA in HNPCC kindreds. Genes Chromosomes Cancer 1999, 26:372-375.

84. Amr S, Heisey C, Zhang M, Xia XJ, Shows KH, Ajlouni K, Pandya A, Satin LS, El-Shanti $H$, Shiang R: A homozygous mutation in a novel zinc-finger protein, ERIS, is responsible for Wolfram syndrome 2. Am J Hum Genet 2007, 81:673-683.

85. Matern D, He M, Berry SA, Rinaldo P, Whitley CB, Madsen PP, van Calcar SC, Lussky RC, Andresen BS, Wolff JA, Vockley J: Prospective diagnosis of 2methylbutyryl-CoA dehydrogenase deficiency in the Hmong population by newborn screening using tandem mass spectrometry. Pediatrics 2003, 112:74-78.

86. Teraoka SN, Telatar M, Becker-Catania S, Liang T, Onengut S, Tolun A, Chessa L, Sanal O, Bernatowska E, Gatti RA, Concannon P: Splicing defects in the ataxia-telangiectasia gene, ATM: underlying mutations and consequences. Am J Hum Genet 1999, 64:1617-1631.

87. Fackenthal JD, Cartegni L, Krainer AR, Olopade OI: BRCA2 T2722R is a deleterious allele that causes exon skipping. Am J Hum Genet 2002, 71:625-631.

88. Houdayer C, Dehainault C, Mattler C, Michaux D, Caux-Moncoutier V, PagesBerhouet S, d'Enghien CD, Lauge A, Castera L, Gauthier-Villars M, StoppaLyonnet D: Evaluation of in silico splice tools for decision-making in molecular diagnosis. Hum Mutat 2008, 29:975-982.

89. Vockley J, Rogan PK, Anderson BD, Willard J, Seelan RS, Smith DI, Liu W: Exon skipping in IVD RNA processing in isovaleric acidemia caused by point mutations in the coding region of the IVD gene. Am J Hum Genet 2000, 66:356-367.

90. Vuillaumier-Barrot S, Barnier A, Cuer M, Durand G, Grandchamp B, Seta N: Characterization of the $415 \mathrm{G}>\mathrm{A}$ (E139K) PMM2 mutation in carbohydrate-deficient glycoprotein syndrome type la disrupting a splicing enhancer resulting in exon 5 skipping. Hum Mutat 1999, 14:543-544.

91. Ohno K, Milone M, Shen XM, Engel AG: A frameshifting mutation in CHRNE unmasks skipping of the preceding exon. Hum Mol Genet 2003, 12:3055-3066

92. Zatkova A, Messiaen L, Vandenbroucke I, Wieser R, Fonatsch C, Krainer AR, Wimmer K: Disruption of exonic splicing enhancer elements is the principal cause of exon skipping associated with seven nonsense or missense alleles of NF1. Hum Mutat 2004, 24:491-501.

93. Mazoyer S, Puget N, Perrin-Vidoz L, Lynch HT, Serova-Sinilnikova OM, Lenoir GM: A BRCA1 nonsense mutation causes exon skipping. Am J Hum Genet 1998, 62:713-715.

94. Ryther RC, Flynt AS, Harris BD, Phillips JA, Patton JG: GH1 splicing is regulated by multiple enhancers whose mutation produces a dominantnegative $\mathrm{GH}$ isoform that can be degraded by allele-specific small interfering RNA (siRNA). Endocrinology 2004, 145:2988-2996.

95. Ozsahin H, Arredondo-Vega FX, Santisteban I, Fuhrer H, Tuchschmid P, Jochum W, Aguzzi A, Lederman HM, Fleischman A, Winkelstein JA, Seger RA, Hershfield MS: Adenosine deaminase deficiency in adults. Blood 1997, 89:2849-2855.

96. Chun K, MacKay N, Petrova-Benedict R, Federico A, Fois A, Cole DE, Robertson $\mathrm{E}$, Robinson $\mathrm{BH}$ : Mutations in the X-linked E1 alpha subunit of pyruvate dehydrogenase: exon skipping, insertion of duplicate sequence, and missense mutations leading to the deficiency of the pyruvate dehydrogenase complex. Am J Hum Genet 1995, 56:558-569.

97. Okajima K, Warman ML, Byrne LC, Kerr DS: Somatic mosaicism in a male with an exon skipping mutation in PDHA1 of the pyruvate dehydrogenase complex results in a milder phenotype. Mol Genet Metab 2006, 87:162-168.

98. Das S, Levinson B, Whitney S, Vulpe C, Packman S, Gitschier J: Diverse mutations in patients with Menkes disease often lead to exon skipping. Am J Hum Genet 1994, 55:883-889.

99. Wang E, Huang Z, Hobson GM, Dimova N, Sperle K, McCullough A, Cambi F: PLP1 alternative splicing in differentiating oligodendrocytes: characterization of an exonic splicing enhancer. J Cell Biochem 2006, 97:999-1016.
100. Valentine CR: The association of nonsense codons with exon skipping. Mutat Res 1998, 411:87-117.

101. Yang $\mathrm{J}$, Lin JG, Hu MC, Wu CW: Mutagenicity and mutational spectrum of N-methyl-N'-nitro-N-nitrosoguanidine in the hprt gene in G1-S and late S phase of diploid human fibroblasts. Cancer Res 1993, 53:2865-2873.

102. Burkhart-Schultz KJ, Thompson $\mathrm{CL}$, Jones IM: Spectrum of somatic mutation at the hypoxanthine phosphoribosyltransferase (hprt) gene of healthy people. Carcinogenesis 1996, 17:1871-1883.

103. Theophilus BD, Enayat MS, Williams MD, Hill FG: Site and type of mutations in the factor VIII gene in patients and carriers of haemophilia A. Haemophilia 2001, 7:381-391.

104. Pagani F, Buratti E, Stuani C, Baralle FE: Missense, nonsense, and neutral mutations define juxtaposed regulatory elements of splicing in cystic fibrosis transmembrane regulator exon 9. J Biol Chem 2003, 278:26580-26588.

105. Elsharawy A, Hundrieser B, Brosch M, Wittig M, Huse K, Platzer M, Becker A, Simon M, Rosenstiel P, Schreiber S, Krawczak M, Hampe J: Systematic evaluation of the effect of common SNPs on pre-mRNA splicing. Hum Mutat 2009, 30:625-632.

106. Lastella P, Resta N, Miccolis I, Quagliarella A, Guanti G, Stella A: Site directed mutagenesis of hMLH1 exonic splicing enhancers does not correlate with splicing disruption. J Med Genet 2004, 41:e72.

107. D'Souza I, Poorkaj P, Hong M, Nochlin D, Lee VM, Bird TD, Schellenberg GD: Missense and silent tau gene mutations cause frontotemporal dementia with parkinsonism-chromosome 17 type, by affecting multiple alternative RNA splicing regulatory elements. Proc Natl Acad Sci USA 1999, 96:5598-5603.

108. Lee VM, Goedert M, Trojanowski JQ: Neurodegenerative tauopathies. Annu Rev Neurosci 2001, 24:1121-1159.

109. Pomponio RJ, Reynolds TR, Mandel H, Admoni O, Melone PD, Buck GA, Wolf $B$ : Profound biotinidase deficiency caused by a point mutation that creates a downstream cryptic 3 ' splice acceptor site within an exon of the human biotinidase gene. Hum Mol Genet 1997, 6:739-745.

110. Asselta R, Duga S, Spena S, Peyvandi F, Castaman G, Malcovati M, Mannucci PM, Tenchini ML: Missense or splicing mutation? The case of a fibrinogen Bbeta-chain mutation causing severe hypofibrinogenemia. Blood 2004, 103:3051-3054

111. Tamouza R, El Kassar N, Schaeffer V, Carbonnelle E, Tatari Z, Marzais F, Fortier C, Poirier JC, Sadki K, Bernaudin F, Toubert A, Krishnamoorthy R, Charron D: A novel HLA-B*39 allele (HLA-B*3916) due to a rare mutation causing cryptic splice site activation. Hum Immunol 2000, 61:467-473.

112. Nakamura K, Fukao T, Perez-Cerda C, Luque C, Song XQ, Naiki Y, Kohno $Y$, Ugarte $\mathrm{M}$, Kondo $\mathrm{N}$ : A novel single-base substitution $(380 \mathrm{C}>\mathrm{T}$ ) that activates a 5-base downstream cryptic splice-acceptor site within exon 5 in almost all transcripts in the human mitochondrial acetoacetyl-CoA thiolase gene. Mol Genet Metab 2001, 72:115-121.

113. Bruce LJ, Ghosh S, King MJ, Layton DM, Mawby WJ, Stewart GW, Oldenborg PA, Delaunay J, Tanner MJ: Absence of CD47 in protein 4.2deficient hereditary spherocytosis in man: an interaction between the Rh complex and the band 3 complex. Blood 2002, 100:1878-1885.

114. Pohlenz J, Rosenthal IM, Weiss RE, Jhiang SM, Burant C, Refetoff S: Congenital hypothyroidism due to mutations in the sodium/iodide symporter. Identification of a nonsense mutation producing a downstream cryptic 3' splice site. J Clin Invest 1998, 101:1028-1035.

115. Tran VK, Takeshima Y, Zhang Z, Habara Y, Haginoya K, Nishiyama A, Yagi M, Matsuo M: A nonsense mutation-created intraexonic splice site is active in the lymphocytes, but not in the skeletal muscle of a DMD patient. Hum Genet 2007, 120:737-742.

116. Frattini A, Orchard PJ, Sobacchi C, Giliani S, Abinun M, Mattsson JP, Keeling DJ, Andersson AK, Wallbrandt P, Zecca L, Notarangelo LD, Vezzoni P, Villa A: Defects in TCIRG1 subunit of the vacuolar proton pump are responsible for a subset of human autosomal recessive osteopetrosis. Nat Genet 2000, 25:343-346.

117. Bourbon $M$, Sun XM, Soutar AK: A rare polymorphism in the low density lipoprotein (LDL) gene that affects mRNA splicing. Atherosclerosis 2007, 195:e17-20.

118. Tavassoli K, Eigel A, Dworniczak B, Valtseva E, Horst J: Identification of four novel mutations in the factor VIII gene: three missense mutations (E1875G, G2088S, I2185T) and a 2-bp deletion (1780delTC). Hum Mutat 1998, , Suppl 1: S260-262. 
119. Reynolds DM, Hayashi T, Cai Y, Veldhuisen B, Watnick TJ, Lens XM Mochizuki T, Qian F, Maeda Y, Li L, Fossdal R, Coto E, Wu G, Breuning MH, Germino GG, Peters DJ, Somlo S: Aberrant splicing in the PKD2 gene as a cause of polycystic kidney disease. J Am Soc Nephrol 1999, 10:2342-2351.

120. Baumgartner MR, Almashanu S, Suormala T, Obie C, Cole RN, Packman S, Baumgartner ER, Valle D: The molecular basis of human 3-methylcrotonylCoA carboxylase deficiency. J Clin Invest 2001, 107:495-504.

121. Verlaan DJ, Siegel AM, Rouleau GA: Krit1 missense mutations lead to splicing errors in cerebral cavernous malformation. Am J Hum Genet 2002, 70:1564-1567.

122. Reifenberger J, Rauch L, Beckmann MW, Megahed M, Ruzicka T, Reifenberger G: Cowden's disease: clinical and molecular genetic findings in a patient with a novel PTEN germline mutation. Br J Dermatol 2003, 148:1040-1046

123. Kaurah P, MacMillan A, Boyd N, Senz J, De Luca A, Chun N, Suriano G, Zaor S, Van Manen L, Gilpin C, Nikkel S, Connolly-Wilson M, Weissman S, Rubinstein WS, Sebold C, Greenstein R, Stroop J, Yim D, Panzini B, McKinnon W, Greenblatt M, Wirtzfeld D, Fontaine D, Coit D, Yoon S, Chung D, Lauwers G, Pizzuti A, Vaccaro C, Redal MA, et al: Founder and recurrent $\mathrm{CDH} 1$ mutations in families with hereditary diffuse gastric cancer. JAMA 2007, 297:2360-2372.

124. Wimmer K, Roca X, Beiglbock H, Callens T, Etzler J, Rao AR, Krainer AR, Fonatsch C, Messiaen L: Extensive in silico analysis of NF1 splicing defects uncovers determinants for splicing outcome upon $5^{\prime}$ splice-site disruption. Hum Mutat 2007, 28:599-612.

125. Ars E, Serra E, Garcia J, Kruyer H, Gaona A, Lazaro C, Estivill X: Mutations affecting mRNA splicing are the most common molecular defects in patients with neurofibromatosis type 1. Hum Mol Genet 2000, 9:237-247.

126. Yang Y, Swaminathan S, Martin BK, Sharan SK: Aberrant splicing induced by missense mutations in BRCA1: clues from a humanized mouse model. Hum Mol Genet 2003, 12:2121-2131.

127. Symoens S, Nuytinck L, Legius E, Malfait F, Coucke PJ, De Paepe A: Met $>$ Val substitution in a highly conserved region of the pro-alpha1(I) collagen C-propeptide domain causes alternative splicing and a mild EDS/OI phenotype. J Med Genet 2004, 41:e96

128. Hashimoto S, Tsukada S, Matsushita M, Miyawaki T, Niida Y, Yachie A, Kobayashi S, Iwata T, Hayakawa H, Matsuoka H, Tsuge I, Yamadori T, Kunikata T, Arai S, Yoshizaki K, Taniguchi N, Kishimoto T: Identification of Bruton's tyrosine kinase (Btk) gene mutations and characterization of the derived proteins in 35 X-linked agammaglobulinemia families: a nationwide study of Btk deficiency in Japan. Blood 1996, 88:561-573.

129. O'Neill JP, Rogan PK, Cariello N, Nicklas JA: Mutations that alter RNA splicing of the human HPRT gene: a review of the spectrum. Mutat Res 1998, 411:179-214

130. Ramesh N, Fuleihan R, Swinton P, Rosen FS, Geha R: A point mutation in exon 2 of the CD40 ligand gene causes the simultaneous expression of two defective mRNA species in X-linked hyperimmunoglobulinemia M. Hum Mol Genet 1995, 4:759-761.

131. Jonsson JJ, Aronovich EL, Braun SE, Whitley CB: Molecular diagnosis of mucopolysaccharidosis type II (Hunter syndrome) by automated sequencing and computer-assisted interpretation: toward mutation mapping of the iduronate-2-sulfatase gene. Am J Hum Genet 1995, 56:597-607.

132. Parrini $E$, Ramazzotti A, Dobyns WB, Mei D, Moro F, Veggiotti $P$, Marini $C$, Brilstra EH, Dalla Bernardina B, Goodwin L, Bodell A, Jones MC, Nangeroni M, Palmeri S, Said E, Sander JW, Striano P, Takahashi Y, Van Maldergem L, Leonardi G, Wright M, Walsh CA, Guerrini R: Periventricular heterotopia: phenotypic heterogeneity and correlation with Filamin A mutations. Brain 2006, 129:1892-1906.

133. Paradisi M, McClintock D, Boguslavsky RL, Pedicelli C, Worman HJ, Djabali K: Dermal fibroblasts in Hutchinson-Gilford progeria syndrome with the lamin A G608G mutation have dysmorphic nuclei and are hypersensitive to heat stress. BMC Cell Biol 2005, 6:27.

134. Dominissini S, Buratti E, Bembi B, Baralle M, Pittis MG: Characterization of two novel GBA mutations causing Gaucher disease that lead to aberrant RNA species by using functional splicing assays. Hum Mutat 2006, 27:119.

135. Baklouti F, Marechal J, Wilmotte R, Alloisio N, Morle L, Ducluzeau MT, Denoroy L, Mrad A, Ben Aribia MH, Kastally R, et al: Elliptocytogenic alpha I/36 spectrin Sfax lacks nine amino acids in helix 3 of repeat 4. Evidence for the activation of a cryptic 5 '-splice site in exon 8 of spectrin alphagene. Blood 1992, 79:2464-2470.
136. Chen W, Kubota S, Teramoto T, Nishimura Y, Yonemoto K, Seyama Y: Silent nucleotide substitution in the sterol 27-hydroxylase gene (CYP 27) leads to alternative pre-mRNA splicing by activating a cryptic 5 ' splice site at the mutant codon in cerebrotendinous xanthomatosis patients. Biochemistry 1998, 37:4420-4428.

137. Gardella R, Zoppi N, Zambruno G, Barlati S, Colombi M: Different phenotypes in recessive dystrophic epidermolysis bullosa patients sharing the same mutation in compound heterozygosity with two novel mutations in the type VII collagen gene. Br J Dermatol 2002, 147:450-457.

138. Denecke J, Kranz C, Kemming D, Koch HG, Marquardt T: An activated 5' cryptic splice site in the human ALG3 gene generates a premature termination codon insensitive to nonsense-mediated mRNA decay in a new case of congenital disorder of glycosylation type Id (CDG-Id). Hum Mutat 2004, 23:477-486.

139. Baumbach L, Schiavi A, Bartlett R, Perera E, Day J, Brown MR, Stein S, Eidson M, Parks JS, Cleveland W: Clinical, biochemical, and molecular investigations of a genetic isolate of growth hormone insensitivity (Laron's syndrome). J Clin Endocrinol Metab 1997, 82:444-451.

140. Berg MA, Guevara-Aguirre J, Rosenbloom AL, Rosenfeld RG, Francke U: Mutation creating a new splice site in the growth hormone receptor genes of 37 Ecuadorean patients with Laron syndrome. Hum Mutat 1992 1:24-32.

141. Yamada S, Tomatsu S, Sly WS, Islam R, Wenger DA, Fukuda S, Sukegawa K, Orii T: Four novel mutations in mucopolysaccharidosis type VII including a unique base substitution in exon 10 of the beta-glucuronidase gene that creates a novel 5'-splice site. Hum Mol Genet 1995, 4:651-655.

142. Suwanmanee T, Sierakowska H, Fucharoen S, Kole R: Repair of a splicing defect in erythroid cells from patients with beta-thalassemia/HbE disorder. Mol Ther 2002, 6:718-726.

143. Goldsmith ME, Humphries RK, Ley T, Cline A, Kantor JA, Nienhuis AW: "Silent" nucleotide substitution in a beta+-thalassemia globin gene activates splice site in coding sequence RNA. Proc Natl Acad Sci USA 1983, 80:2318-2322.

144. Hospach $T$, Lohse $P$, Heilbronner $H$, Dannecker GE, Lohse $P$ : Pseudodominant inheritance of the hyperimmunoglobulinemia D with periodic fever syndrome in a mother and her two monozygotic twins. Arthritis Rheum 2005, 52:3606-3610.

145. Cockerill FJ, Hawa NS, Yousaf N, Hewison M, O'Riordan JL, Farrow SM: Mutations in the vitamin $D$ receptor gene in three kindreds associated with hereditary vitamin D resistant rickets. J Clin Endocrinol Metab 1997 82:3156-3160.

146. Wicklow BA, Ivanovich JL, Plews MM, Salo TJ, Noetzel MJ, Lueder GT, Cartegni L, Kaback MM, Sandhoff K, Steiner RD, Triggs-Raine BL: Severe subacute GM2 gangliosidosis caused by an apparently silent HEXA mutation (V324V) that results in aberrant splicing and reduced HEXA mRNA. Am J Med Genet A 2004, 127A:158-166.

147. Harteveld CL, Wijermans PW, van Delft P, Rasp E, Haak HL, Giordano PC: An alpha-thalassemia phenotype in a Dutch Hindustani, caused by a new point mutation that creates an alternative splice donor site in the first exon of the alpha2-globin gene. Hemoglobin 2004, 28:255-259.

148. Lavery GG, Ronconi V, Draper N, Rabbitt EH, Lyons V, Chapman KE, Walker EA, McTernan CL, Giacchetti G, Mantero F, Seckl JR, Edwards CR, Connell JM, Hewison M, Stewart PM: Late-onset apparent mineralocorticoid excess caused by novel compound heterozygous mutations in the HSD11B2 gene. Hypertension 2003, 42:123-129.

149. Xie J, Pabon D, Jayo A, Butta N, Gonzalez-Manchon C: Type I Glanzmann thrombasthenia caused by an apparently silent beta3 mutation that results in aberrant splicing and reduced beta3 mRNA. Thromb Haemost 2005, 93:897-903.

150. Candotti F, Oakes SA, Johnston JA, Giliani S, Schumacher RF, Mella P, Fiorini M, Ugazio AG, Badolato $R$, Notarangelo LD, Bozzi F, Macchi $P$, Strina D, Vezzoni P, Blaese RM, O'Shea JJ, Villa A: Structural and functional basis for JAK3-deficient severe combined immunodeficiency. Blood 1997, 90:3996-4003.

151. Dale DC, Person RE, Bolyard AA, Aprikyan AG, Bos C, Bonilla MA, Boxer LA, Kannourakis G, Zeidler C, Welte K, Benson KF, Horwitz M: Mutations in the gene encoding neutrophil elastase in congenital and cyclic neutropenia. Blood 2000, 96:2317-2322.

152. Yip KL, Chan SY, Ip WK, Lau YL: Bruton's tyrosine kinase mutations in 8 Chinese families with X-linked agammaglobulinemia. Hum Mutat 2000, 15:385. 
153. Donati MA, Malvagia S, Pasquini E, Morrone A, La Marca G, Garavaglia B,

Toniolo D, Zammarchi E: Barth syndrome presenting with acute

metabolic decompensation in the neonatal period. J Inherit Metab Dis 2006, 29:684.

154. Flomen RH, Green PM, Bentley DR, Giannelli F, Green EP: Detection of point mutations and a gross deletion in six Hunter syndrome patients. Genomics 1992, 13:543-550.

doi:10.1186/gb-2010-11-2-r20

Cite this article as: Woolfe et al: Genomic features defining exonic variants that modulate splicing. Genome Biology 2010 11:R20.

Submit your next manuscript to BioMed Central and take full advantage of:

- Convenient online submission

- Thorough peer review

- No space constraints or color figure charges

- Immediate publication on acceptance

- Inclusion in PubMed, CAS, Scopus and Google Scholar

- Research which is freely available for redistribution

Submit your manuscript at www.biomedcentral.com/submit 